Pacific Journal of Mathematics

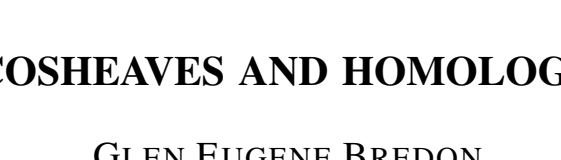




\title{
COSHEAVES AND HOMOLOGY
}

\author{
GLEN E. BREDON
}

In this paper we initiate a study of the theory of cosheaves of modules. We are interested mainly in those facts which are not encompassed by the known theories of sheaves with values in general categories. A central result is the establishment of the existence of a reasonably large subcategory of the category of precosheaves and a reflector from this to the subcategory of cosheaves. The general theory is applied to the study of the Čech, singular, and Borel-Moore homology theories. The main applications establish that the Čech and Borel-Moore homology theories coincide on locally compact and paracompact $c l c^{\infty}$ spaces and that the Čech and singular theories coincide on paracompact HLC spaces. These isomorphisms are established for locally constant coefficients. For constant coefficients the latter result was originally established by Mardešic and the former by 0 . Jussila. There are also applications to acyclic coverings and to mappings.

There have been several treatments of sheaf theory with values in an arbitrary category, notably [6] and [9]. More accurately these treatments can be described as attempts to delineate the types of value categories for which the theory goes through in reasonably close analogy to the classical sheaf theory (values in the category of sets, of modules, etc.). Little or no attempt has been made to develop the theory in categories which will not admit such a close analogy. To our mind the most interesting and important nonclassical case is that of cosheaves of $L$-modules; that is, of sheaves with values in the dual category to the category of $L$-modules (this may be regarded as the category of compact $L$-modules; see [9, p. 77]). Unfortunately the theories of [6] and [9] are hopelessly inadequate for this case. [For example, in [6] it is assumed that the value category for sheaf theory has enough "small" objects (termed "very small" in [9]), but it is easily seen that the only cosmall object in the category of $L$ modules is zero, for any ring $L$.]

From now on we shall let $L$ denote a given base ring with unity and the terms cosheaf, precosheaf, sheaf, presheaf, etc. will always presuppose the category of $L$-modules as the value category. Categorical terminology will be that of [9]. "Covering" always means an open covering.

In [1, Chap. V] the notion of cosheaves was put to work to obtain some deep results on homology theory. However, we did not attempt there to give a coherent "theory" of cosheaves, outside of some results 
necessary to our immediate purposes and of some indications in the exercises.

In this paper we shall initiate such a study. In our opinion one cannot expect to find anything like a complete duality with sheaf theory and one must be prepared, from the start, to dispense with some basic properties. The most basic concept in sheaf theory is that of a sheaf generated by a given presheaf. In categorical terminology [9] this is the concept of a reflector from presheaves to sheaves. We believe that there is not much hope for the existence of a reflector from precosheaves to cosheaves. However, we shall obtain such a reflector on a reasonably large subcategory of precosheaves containing the cosheaves and, on locally connected spaces, the constant precosheaves.

The general theory of cosheaves, together with material on Čech homology, occupies the first five sections of the paper. In later sections we apply parts of this general theory (but not all of it by any means) to the study of the relationships between the Čech, singular, and Borel-Moore homology theories. These applications complement the results of [1, Chap. V] on the relationships between the singular and Borel-Moore homology theories, but the present treatment is largely independent of that work.

Some generalities on cosheaves may be found in [2], but the contact with the present paper is negligible.

1. Cosheaves. Let $X$ be a topological space. A precosheaf $\mathfrak{A}$ (of $L$-modules) on $X$ is a covariant functor from the category of open sets in $X$ and inclusions to the category of $L$-modules. For $U \subset V$ the corresponding map $\mathfrak{U}(U) \rightarrow \mathfrak{U}(V)$ is denoted by $i_{V, U}$.

A precosheaf $\mathfrak{A}$ on $X$ is called a cosheaf if, for every covering $\left\{U_{i}\right\}$ of an open set $U \subset X$, the sequence

$$
\underset{\langle i, j\rangle}{\bigoplus_{1}} \mathfrak{A}\left(U_{i} \cap U_{j}\right) \stackrel{\beta}{\longrightarrow} \bigoplus_{i} \mathfrak{A}\left(U_{i}\right) \stackrel{\alpha}{\longrightarrow} \mathfrak{A}(U) \longrightarrow 0
$$

is exact, where $\alpha=\sum_{i} i_{U, U_{i}}$ and $\beta=\sum_{\langle i, j\rangle}\left(i_{U_{i}, U_{i} \cap U_{j}}-i_{U_{j}, U_{i} \cap U_{j}}\right)$. If we only require $\alpha$ to be epimorphic then $\mathfrak{A}$ is called an epiprecosheaf (the dual notion is that of a monopresheaf; see [9, p. 246]).

The following result shows that the cokernel of a homomorphism of cosheaves is a cosheaf.

Proposition 1.1. Let $\mathfrak{A} \rightarrow \mathfrak{A} \rightarrow \mathfrak{A}^{\prime \prime} \rightarrow 0$ be an exact sequence of precosheaves. If $\mathfrak{U}^{\prime}$ is an epiprecosheaf and $\mathfrak{A}$ is a cosheaf then $\mathfrak{U}^{\prime \prime}$ is a cosheaf.

Proof. This follows from elementary diagram chasing and is also 
valid for values in any abelian category (see [9; p. 254]). The details will be omitted. Similar remarks apply to the following result.

Proposition 1.2. Let $0 \rightarrow \mathfrak{U} \rightarrow \mathfrak{U} \rightarrow \mathfrak{U}^{\prime \prime} \rightarrow 0$ be an exact sequence of precosheaves. If $\mathfrak{U}$ is an epiprecosheaf and $\mathfrak{U}^{\prime \prime}$ is a cosheaf then $\mathfrak{H}^{\prime}$ is an epiprecosheaf.

Proposition 1.3. Let $\mathfrak{U}$ be a precosheaf. Then $\mathfrak{X}$ is a cosheaf if and only if the following two conditions are satisfied:

( a ) $\mathfrak{H}(U \cap V) \stackrel{\beta}{\longrightarrow} \mathfrak{Y}(U) \oplus \mathfrak{X}(V) \stackrel{\alpha}{\longrightarrow} \mathfrak{P}(U \cup V) \rightarrow 0$ is exact for all open $U$ and $V$ where $\beta=\left(i_{U, U \cap V}-i_{V, U \cap V}\right)$ and $\alpha=i_{U \cup V, U}+i_{U \cup V, V}$.

(b) If $\left\{U_{i}\right\}$ is directed upwards by inclusion then the natural map $\lim \mathfrak{Y}\left(U_{i}\right) \rightarrow \mathfrak{Y}\left(\cup U_{i}\right)$ is an isomorphism.

Proof. It clearly suffices to prove that if (a) is satisfied, then for any finite collection $\left\{U_{0}, U_{1}, \cdots, U_{n}\right\}$ the sequence

$$
\underset{\langle i}{\bigoplus_{j}} \mathfrak{A}\left(U_{i} \cap U_{j}\right) \stackrel{\beta}{\longrightarrow} \bigoplus_{i} \mathfrak{U}\left(U_{i}\right) \stackrel{\alpha}{\longrightarrow} \mathfrak{U}(U) \longrightarrow 0
$$

is exact, where $U=U U_{i}$. Exactness on the right is clear by an easy induction. The proof of exactness in the middle will be by induction on $n$. Let $U^{\prime}=U_{1} \cup \cdots \cup U_{n}$ and $V=U_{0} \cap U^{\prime}$. Let $s_{j} \in \mathfrak{U}\left(U_{j}\right) j=0,1, \cdots, n$ be such that $\sum_{0}^{n} i_{U, U_{j}}\left(s_{j}\right)=0$ in $\mathfrak{U}(U)$. Let $t^{\prime}=\sum_{1}^{n} i_{U^{\prime}, U_{j}}\left(s_{j}\right) \in \mathfrak{U}\left(U^{\prime}\right)$. Then $i_{U, U_{0}}\left(s_{0}\right)+i_{U, U}\left(t^{\prime}\right)=0$ so that by (a) there exists a $v \in \mathfrak{Y}\left(U_{0} \cap U^{\prime}\right)=\mathfrak{X}(V)$ with

$$
i_{U_{0}, V}(v)=s_{0}, i_{U^{\prime}, V}(v)=-t^{\prime} .
$$

Now $V=\left(U_{0} \cap U_{1}\right) \cup \cdots \cup\left(U_{0} \cap U_{n}\right)$ so that there exist $v_{j} \in \mathfrak{P}\left(U_{0} \cap U_{j}\right)$ for $1 \leqq j \leqq n$ with

$$
v=\sum_{i}^{n} i_{V, U_{0} \cap U_{j}}\left(v_{j}\right)
$$

Thus

$$
\beta\left(\bigoplus_{\langle 0, j\rangle} v_{j}\right)=\left\langle\sum_{1}^{n} i_{U_{0}, U_{0} \cap U_{j}}\left(v_{j}\right),-i_{U_{1}, U_{0} \cap U_{1}}\left(v_{1}\right), \cdots,-i_{U_{n}, U_{0} \cap U_{n}}\left(v_{n}\right)\right\rangle
$$

and an easy computation shows that the element

$$
\bigoplus_{i=0}^{n} s_{i}-\beta\left(\bigoplus_{\langle 0 . j\rangle} v_{j}\right) \in \bigoplus \mathfrak{U}\left(U_{i}\right)
$$

has zero component in $\mathfrak{X}\left(U_{0}\right)$ and projects to zero in $\mathfrak{A}\left(U_{1} \cup \cdots \cup U_{n}\right)$. Thus the result follows from the inductive hypothesis.

A cosheaf $\mathfrak{A}$ is said to be flabby if each $i_{V, U}: \mathfrak{A}(U) \rightarrow \mathfrak{A}(V)$ is a 
monomorphism. [Note that in [1, V.1] it is shown that, for $X$ locally compact, the flabby cosheaves are precisely the cosheaves of sections with compact supports of $c$-soft sheaves. No corresponding characterization is known on nonlocally compact spaces.]

The following result is basic:

Proposition 1.4. Let

$$
0 \rightarrow \mathfrak{\mathfrak { X } ^ { \prime }} \stackrel{f}{\longrightarrow} \mathfrak{X} \stackrel{g}{\longrightarrow} \mathfrak{H}^{\prime \prime} \rightarrow 0
$$

be an exact sequence of precosheaves. Assume that $\mathfrak{A}$ is a cosheaf and that $\mathfrak{U}^{\prime \prime}$ is a flabby cosheaf. Then $\mathfrak{V}^{\prime}$ is a cosheaf.

Proof. We will verify (a) and (b) of (1.3) for $\mathfrak{Y}^{\prime}$. Part (b) is an immediate consequence of the exactness of the direct limit functor. Part (a) follows from a diagram chase in

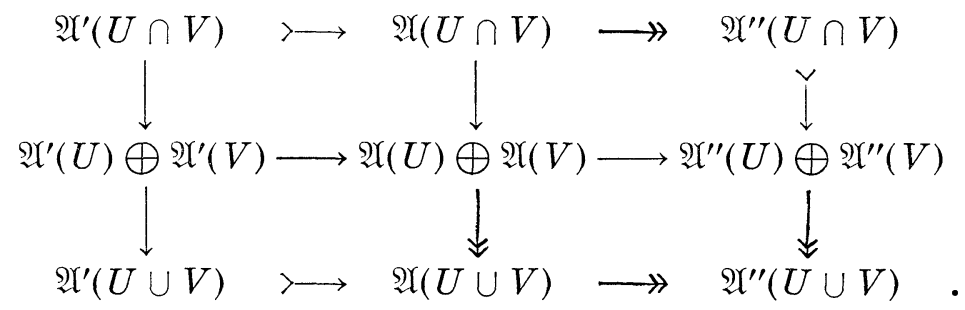

Proposition 1.5. Let $\mathfrak{A}$ be a precosheaf. The class of subprecosheaves of $\mathfrak{A}$ which are epiprecosheaves contains a maximum element which is denoted by $\widetilde{\mathfrak{A}}$. Any homomorphism $\mathfrak{B} \rightarrow \mathfrak{A}$ with $\mathfrak{B}$ an epiprecosheaf factors through $\widetilde{\mathfrak{A}}$.

Proof. This follows from results of the general theory of categories (see [9, p. 131]). For a direct proof, we define by transfinite induction:

$$
\begin{aligned}
\mathfrak{A}_{0}= & \mathfrak{A} \\
\mathfrak{A}_{\alpha+1}(U)= & \left\{s \in \mathfrak{A}_{\alpha}(U) \mid s \in \operatorname{Im}\left(\bigoplus \mathfrak{A}_{\alpha}\left(U_{i}\right) \longrightarrow \mathfrak{U}_{\alpha}(U)\right)\right. \\
& \text { for each covering } \left.\left\{U_{i}\right\} \text { of } U\right\} \\
\mathfrak{A}_{\beta}(U)= & \bigcap_{\alpha<\beta} \mathfrak{N}_{\alpha}(U) \text { for } \beta \text { a limit ordinal. }
\end{aligned}
$$

Then clearly there exists an ordinal $\alpha$ with $\mathfrak{A}_{\alpha+1}=\mathfrak{A}_{\alpha}$ and we let $\widetilde{\mathfrak{A}}=\mathfrak{A}_{\alpha}$. The properties claimed are clear from this construction.

Note that the last statement implies that every homomorphism $\mathfrak{A} \rightarrow \mathfrak{B}$ restricts to a homomorphism $\widetilde{\mathfrak{U}} \rightarrow \widetilde{\mathfrak{B}}$ and hence $\mathfrak{N} \mapsto \widetilde{\mathfrak{U}}$ is a functor. 
2. Local triviality. As in $[1, p .219]$ we say that a precosheaf $\mathfrak{A}$ is locally zero if for each open $U \subset X$ and $x \in U$ there is a smaller neighborhood $V$ of $x$ with $i_{U, V}: \mathfrak{A}(V) \rightarrow \mathfrak{A}(U)$ zero. Clearly an epiprecosheaf is locally zero if and only if it is zero.

A sequence $\mathfrak{A}^{\prime} \stackrel{f}{\longrightarrow} \mathfrak{A} \stackrel{g}{\longrightarrow} \mathfrak{X}^{\prime \prime}$ of precosheaves will be said to be locally exact if $g \circ f=0$ and if the precosheaf $\operatorname{Ker} g / \operatorname{Im} f$ is locally zero. By exactness of a sequence of cosheaves we mean exactness as a sequence of precosheaves.

Proposition 2.1. The sequence $\mathfrak{X}^{\prime} \stackrel{f}{\longrightarrow} \mathfrak{A} \stackrel{g}{\longrightarrow} \mathfrak{X}^{\prime \prime} \rightarrow 0$ of cosheaves is locally exact if and only if it is exact. (It suffices that $\mathfrak{U}^{\prime \prime}$ is a cosheaf and $\mathfrak{X}$ is an epiprecosheaf.)

Proof. Coker $g$ is a cosheaf by (1.1) and, since it is locally zero by hypothesis, it must be zero. This proves exactness at $\mathfrak{A}^{\prime \prime}$. By (1.2) Ker $g$ is an epiprecosheaf and it follows immediately that $\operatorname{Ker} g / \operatorname{Im} f$ is an epiprecosheaf. Since $\operatorname{Ker} g / \operatorname{Im} f$ is locally zero it must be zero, so that $\operatorname{Im} f=\operatorname{Ker} g$.

Proposition 2.2. If $0 \rightarrow \mathfrak{X ^ { \prime }} \stackrel{f}{\longrightarrow} \mathfrak{A} \stackrel{g}{\longrightarrow} \mathfrak{A}^{\prime \prime} \rightarrow 0$ is a locally exact sequence of cosheaves with $\mathfrak{A}^{\prime \prime}$ flabby then this sequence is exact. If $\mathfrak{A}$ is also flabby then so is $\mathfrak{A}^{\prime}$.

Proof. This sequence is exact at $\mathfrak{U}$ and at $\mathfrak{U}^{\prime \prime}$ by (2.1). By (1.4), $\operatorname{Ker} g=\operatorname{Im} f$ is a cosheaf. Clearly $0 \rightarrow \mathfrak{A X}^{\prime} \rightarrow \operatorname{Im} f \rightarrow 0$ is locally exact and hence exact by (2.1). The last statement follows from an easy diagram chase.

CoRollary 2.3. If

$$
\mathfrak{I}_{N} \stackrel{d_{N}}{\longrightarrow} \cdots \longrightarrow \mathfrak{A}_{2} \stackrel{d_{2}}{\longrightarrow} \mathfrak{A}_{1} \stackrel{d_{1}}{\longrightarrow} \mathfrak{A}_{0} \longrightarrow 0
$$

is a locally exact sequence of flabby cosheaves, then it is exact and $\operatorname{Ker} d_{N}$ is a flabby cosheaf.

$$
\begin{array}{ll}
\text { Proof. } & \text { Let } \mathfrak{Z}_{n}=\operatorname{Ker} d_{n} \text {. Clearly } \\
& \cdots \longrightarrow \mathfrak{A}_{n+3} \longrightarrow \mathfrak{A}_{n+2} \longrightarrow \mathfrak{A}_{n+1} \longrightarrow \mathfrak{Z}_{n} \longrightarrow 0
\end{array}
$$

is locally exact. By (2.1)

$$
0 \longrightarrow \mathfrak{B}_{1} \longrightarrow \mathfrak{A}_{1} \longrightarrow \mathfrak{A}_{0} \longrightarrow 0
$$

is exact. By (1.4) $3_{1}$ is a cosheaf and, by (2.2), it is flabby. By induction we see that each $3_{n}$ is a flabby cosheaf and that each sequence 


$$
0 \longrightarrow 3_{n} \longrightarrow \mathfrak{A}_{n} \stackrel{\cdot}{\longrightarrow} 3_{n-1} \longrightarrow 0
$$

is exact. The result follows.

LEмма 2.4. The class of locally zero precosheaves is closed under formation of subprecosheaves, quotient precosheaves, and extensions.

Proof. All three parts may be handled simultaneously. Let $\mathfrak{A}^{\prime} \rightarrow \mathfrak{A} \rightarrow \mathfrak{Y}^{\prime \prime}$ be exact with $\mathfrak{X}^{\prime}$ and $\mathfrak{U}^{\prime \prime}$ locally zero. Let $U$ be open and $x \in U$. Let $V \subset U$ be a neighborhood of $x$ such that $\mathfrak{A}^{\prime \prime}(V) \rightarrow$ $\mathfrak{A}^{\prime \prime}(U)$ is trivial and let $W \subset V$ be a neighborhood of $x$ such that $\mathfrak{U}^{\prime}(W) \rightarrow \mathfrak{U}^{\prime}(V)$ is trivial. Diagram chasing in

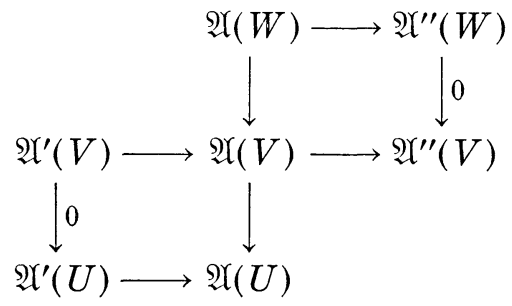

shows that $\mathfrak{A}(W) \rightarrow \mathfrak{A}(U)$ is zero as desired. (The cases of subprecosheaves and quotient precosheaves are given by taking $\mathfrak{U}^{\prime}=0$ or $\mathfrak{A}^{\prime \prime}=0$ respectively.)

REMARK 2.5. We are grateful to the referee for pointing out that (2.4) shows that the locally zero precosheaves form a "thick subcategory" (see [4, p. 15]). If one passes to the quotient category then the local isomorphisms (below) and locally exact sequences become actual isomorphisms and exact sequences. Clearly some of the propositions in the next section can be regarded as special cases of general facts about such quotient categories.

3. Local isomorphisms. A homomorphism $h: \mathfrak{A} \rightarrow \mathfrak{B}$ between two precosheaves is said to be a local isomorphism if Ker $h$ and Coker $h$ are locally zero (see $[1 ;$ p. 219]).

If $\mathfrak{A}$ is an epiprecosheaf and $\mathfrak{B}$ is a cosheaf then a local isomorphism $h: \mathfrak{A} \rightarrow \mathfrak{B}$ is necessarily an isomorphism by (1.1) and (1.2).

Consider commutative diagrams of precosheaves of the form

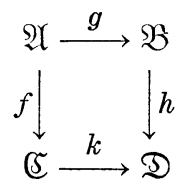


Proposition 3.2. If (3.1) is a pusbout diagram and if $f$ is a local isomorphism then so is $h$. Dually, if (3.1) is a pullback diagram and if $h$ is a local isomorphism then so is $f$.

Proof. We shall only give the proof for the second part since both parts are analogous. If (3.1) is a pullback then we may assume that $\mathfrak{U}$ is given by

$$
\mathfrak{A}(U)=\{(b, c) \in \mathfrak{B}(U) \times \mathfrak{E}(U) \mid h(b)=k(c)\} .
$$

Moreover $g$ and $f$ are given by the projections on the first and second factors respectively. We see that $\operatorname{Ker} f=\{(b, 0) \mid h(b)=0\}$ so that

$$
0 \longrightarrow \operatorname{Ker} f \stackrel{g}{\longrightarrow} \operatorname{Ker} h
$$

is exact. Similarly, if $c \in \mathfrak{C}(U)$ and if $k(c) \in \operatorname{Im} h$ then $c \in \operatorname{Im} f$, and it follows that

$$
0 \longrightarrow \text { Coker } f \stackrel{k}{\longrightarrow} \text { Coker } h
$$

is exact. The contention follows from (2.4) applied to the exact sequences (3.3) and (3.4).

CoROLlary 3.5. Let $\mathfrak{B}$ and $\mathbb{E}$ be precosheaves. Then the following statements are equivalent:

(a) There exist a precosheaf $\mathfrak{X}$ and local isomorphisms $\mathfrak{B} \leftarrow$ $\mathfrak{A} \rightarrow \mathfrak{E}$.

(b) There exist a precosheaf $\mathfrak{D}$ and local isomorphisms $\mathfrak{B} \rightarrow$ D)〔(

If one, hence both, of the conditions in (3.5) are satisfied then $\mathfrak{B}$ and $\mathbb{E}$ are said to be equivalent. It is an easy consequence of (3.5) and the following lemma that "equivalence" is an equivalence relation.

LeMma 3.6. Composites of local isomorphisms are local isomorphisms.

Proof. Suppose $\mathfrak{A} \stackrel{f}{\longrightarrow} \mathfrak{B} \stackrel{g}{\longrightarrow}$ (5 are local isomorphisms. Clearly we have the exact sequences

$$
\begin{gathered}
0 \longrightarrow \operatorname{Ker} f \longrightarrow \operatorname{Ker} g f \stackrel{f}{\longrightarrow} \operatorname{Ker} g \\
\text { Coker } f \stackrel{g}{\longrightarrow} \text { Coker } g f \longrightarrow \text { Coker } g \longrightarrow 0
\end{gathered}
$$

and the contention follows immediately from (2.4).

As we have remarked above, locally isomorphic cosheaves are 
isomorphic. It is not so clear that this is also true for equivalent cosheaves, but we shall, in fact, prove this fact later, in (5.7).

Definition 3.7. A precosheaf is said to be smooth if it is equivalent to a cosheaf.

We shall show later that for any smooth precosheaf $\mathfrak{A}$ there is an associated cosheaf $\Phi(\mathfrak{A})$ (unique to isomorphism) and a local isomorphism $\Phi(\mathfrak{U}) \rightarrow \mathfrak{U}$. Also the functor $\Phi$ taking a smooth precosheaf into this "associated cosheaf" will be shown to be a reflector from the category of smooth precosheaves to that of cosheaves. For these reasons one might prefer to substitute the terms "copresheaf" and "precosheaf" for what we call "precosheaf" and "smooth precosheaf" respectively, but we shall not adopt that terminology.

Proposition 3.8. Suppose that we have a commutative diagram

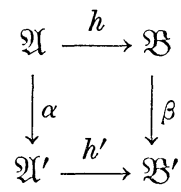

of precosheaves such that $\alpha$ and $\beta$ are local isomorphisms. Then the induced maps $\operatorname{Ker} h \rightarrow \operatorname{Ker} h^{\prime}, \operatorname{Im} h \rightarrow \operatorname{Im} h^{\prime}$, and Coker $h \rightarrow$ Coker $h^{\prime}$ are all local isomorphisms.

Proof. Denote kernels, images, and cokernels by $\Re, \Im$, and $\mathfrak{5}$ respectively. Then we have the commutative diagrams
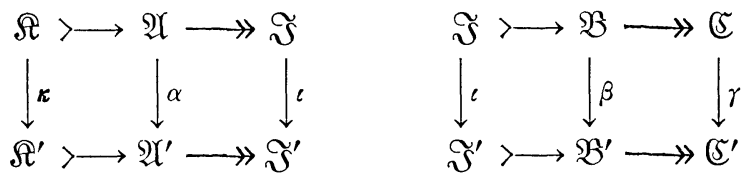

which induce the exact sequences

$$
\begin{aligned}
& 0 \rightarrow \operatorname{Ker} \kappa \rightarrow \operatorname{Ker} \alpha \rightarrow \operatorname{Ker} \iota \rightarrow \text { Coker } \kappa \rightarrow \text { Coker } \alpha \rightarrow \text { Coker } \iota \rightarrow 0 \\
& 0 \rightarrow \operatorname{Ker} \iota \rightarrow \operatorname{Ker} \beta \rightarrow \operatorname{Ker} \gamma \rightarrow \text { Coker } \iota \rightarrow \text { Coker } \beta \rightarrow \text { Coker } \gamma \rightarrow 0 .
\end{aligned}
$$

By hypothesis we have that $\operatorname{Ker} \alpha, \operatorname{Ker} \beta, \operatorname{Coker} \alpha$, and $\operatorname{Coker} \beta$ are locally zero. It follows that $\operatorname{Ker} \kappa, \operatorname{Ker} \iota$, Coker $\iota$, and Coker $\gamma$ are locally zero and that $\operatorname{Ker} \iota \rightarrow \operatorname{Coker} \kappa$ and $\operatorname{Ker} \gamma \rightarrow$ Coker $\iota$ are local isomorphisms. Since Ker $\iota$ and Coker $\iota$ are locally zero it follows from (2.4) that their local isomorphs Coker $\kappa$ and $\operatorname{Ker} \gamma$ are also locally zero. This proves the proposition. 
CoRollary 3.9. Suppose we have a commutative diagram

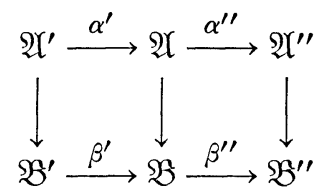

of precosheaves in which the verticals are local isomorphisms and the compositions in the rows are zero. Then the induced map of precosheaves

$\operatorname{Ker} \alpha^{\prime \prime} / \operatorname{Im} \alpha^{\prime} \longrightarrow \operatorname{Ker} \beta^{\prime \prime} / \operatorname{Im} \beta^{\prime}$

is a local isomorphism.

REMARK 3.10. The reader should note that the analogues, in sheaf theory, of the concepts "locally zero" and "local isomorphism" are somewhat stronger conditions than the concepts which usually are described by those terms. The exact analogues of the usual sheaftheoretic concepts are, in our opinion, inadequate for the attainment of most practical aims in cosheaf theory, a fact which is traceable to the unpleasantness of inverse limits.

4. Čech homology. Let $\mathfrak{A}$ be a precosheaf on a space $X$ and let $\mathfrak{U}=\left\{U_{i}\right\}$ be any (open) covering of $X$. We define the group of Čech $p$-chains of the covering $\mathfrak{U}$ to be

$$
\check{C}_{p}(\mathfrak{U} ; \mathfrak{U})=\bigoplus \mathfrak{A}\left(U\left(i_{0}, \cdots, i_{p}\right)\right)
$$

where the sum ranges over all ordered $(p+1)$-tuples $\left\langle i_{0}, \cdots, i_{p}\right\rangle$ of indices and $U\left(i_{0}, \cdots, i_{p}\right)$ stands for $U_{i_{0}} \cap U_{i_{1}} \cap \cdots \cap U_{i_{p}}$. Note that this is an exact functor of precosheaves $\mathfrak{A}$. The differential

$$
\partial: \check{C}_{p}(\mathfrak{U} ; \mathfrak{A}) \longrightarrow \check{C}_{p-1}(\mathfrak{H} ; \mathfrak{A})
$$

is defined by $\partial=\sum_{\jmath=0}^{p}(-1)^{j} \lambda_{j}$ where $\lambda_{j}$ is the canonical map

$$
\mathfrak{A}\left(U\left(i_{0}, \cdots, i_{p}\right)\right) \longrightarrow \mathfrak{A}\left(U\left(i_{0}, \cdots, \hat{i}_{j}, \cdots, i_{p}\right)\right) \text {. }
$$

We also have the augmentation

$$
\varepsilon: \check{C}_{0}(\mathfrak{U} ; \mathfrak{A}) \longrightarrow \mathfrak{U}(X)
$$

given by $\varepsilon=\sum i_{X, U j}: \bigoplus_{j} \mathfrak{A}\left(U_{j}\right) \rightarrow \mathfrak{A}(X)$.

As usual the homology of the chain complex $\check{C}_{*}(\mathfrak{U}$; $\mathfrak{A})$ is denoted by

$$
\check{H}_{*}(\mathfrak{U} ; \mathfrak{X}) \text {, }
$$


and $\varepsilon$ induces a homomorphism

$$
\varepsilon_{*}: \check{H}_{0}(\mathfrak{U} ; \mathfrak{A}) \longrightarrow \mathfrak{U}(X) \text {. }
$$

For an open set $V \subset X, \mathfrak{u} \cap V$ denotes the covering $\left\{U_{i} \cap V\right\}$ of $V$. Thus

$$
V \longmapsto \check{H}_{n}(\mathfrak{U} \cap V ; \mathfrak{U})
$$

defines a precosheaf on $X$ denoted by $\breve{\mathfrak{S}}_{n}(\mathfrak{U} ; \mathfrak{A})$ and

$$
\varepsilon_{*}: \check{\mathfrak{E}}_{n}(\mathfrak{U} ; \mathfrak{A}) \longrightarrow \mathfrak{U}
$$

is a homomorphism of precosheaves. Note that $\varepsilon_{*}$ is epimorphic for all $\mathfrak{U}$ if $\mathfrak{U}$ is an epiprecosheaf and that $\varepsilon_{*}$ is isomorphic for all $\mathfrak{U}$ if $\mathfrak{U}$ is a cosheaf.

If $\mathfrak{B}$ is a refinement of $\mathfrak{U}$ and $\mathfrak{B} \rightarrow \mathfrak{U}$ is a refinement projection then there is an induced chain map $\check{C}_{*}(\mathfrak{B} ; \mathfrak{U}) \rightarrow \check{C}_{*}(\mathfrak{U} ; \mathfrak{U})$ and the induced map $\check{H}_{*}(\mathfrak{B} ; \mathfrak{A}) \rightarrow \check{H}_{*}(\mathfrak{U} ; \mathfrak{A})$ is independent of the refinement projection. (The reader map supply the details of these well-known facts.) The Čech homology of $X$ with coefficients in the precosheaf $\mathfrak{A}$ is defined by

$$
\check{H}_{*}(X ; \mathfrak{U})=\lim _{\longleftarrow} \check{H}_{*}(\mathfrak{U} ; \mathfrak{A})
$$

(the limit taken over all coverings of $X$ ). Clearly

$$
{\breve{\mathfrak{C}_{n}}}(X ; \mathfrak{A}): U \longmapsto \breve{H}_{n}(U ; \mathfrak{A})
$$

is a precosheaf and there is the augmentation homomorphism

$$
\varepsilon_{*}: \check{\mathfrak{E}}_{0}(X ; \mathfrak{A}) \longrightarrow \mathfrak{A}
$$

which is an isomorphism when $\mathfrak{A}$ is a cosheaf.

For a covering $\mathfrak{U}$ of $X$, the precosheaf

$$
V \longmapsto \check{C}_{n}(\mathfrak{U} \cap V ; \mathfrak{U})
$$

will be denoted by $\breve{\mathfrak{C}}_{n}(\mathfrak{U} ; \mathfrak{U})$.

LEMMA 4.1. If $\mathfrak{A}$ is a cosheaf then so is $\check{\mathfrak{E}}_{n}(\mathfrak{U} ; \mathfrak{A})$. The latter is flabby when $\mathfrak{A}$ is flabby.

Proof. The reader may show that the direct sum of a family of cosheaves is a cosheaf. But $\breve{\mathfrak{G}}_{n}(\mathfrak{U} ; \mathfrak{\mathfrak { U }})$ is the direct sum of precosheaves of the form

$$
V \longmapsto \mathfrak{A}(U \cap V)
$$


(where $U=U\left(i_{0}, \cdots, i_{n}\right)$ ) and these are easily seen to be cosheaves when $\mathfrak{A}$ is a cosheaf. The last statement follows easily from similar reasoning.

THEOREM 4.2. For a precosheaf $\mathfrak{A}$ the sequence

$$
\ldots \longrightarrow \breve{\mathfrak{E}}_{2}(\mathfrak{U} ; \mathfrak{U}) \longrightarrow \breve{\mathfrak{C}}_{1}(\mathfrak{U} ; \mathfrak{N}) \longrightarrow \breve{\mathfrak{C}}_{0}(\mathfrak{U} ; \mathfrak{U}) \longrightarrow \mathfrak{A} \longrightarrow 0
$$

of precosheaves is locally exact for any covering $\mathfrak{U}$ of $X$.

Proof. It is clearly sufficient to prove that the sequence is exact when $X$ is a member of $\mathfrak{U}$ since this condition is realized upon restriction to any member of $\mathfrak{u}$. We shall in fact provide a splitting under these circumstances. When $X \in \mathfrak{U}$ define $D: \check{C}_{n}(\mathfrak{U} ; \mathfrak{A}) \rightarrow \check{C}_{n+1}(\mathfrak{U} ; \mathfrak{A})$ by the “identity” $\mathfrak{A}\left(U_{i_{0}} \cap \cdots \cap U_{i_{n}}\right) \rightarrow \mathfrak{A}\left(X \cap U_{i_{0}} \cap \cdots \cap U_{i_{n}}\right)$. (The naturality of this definition provides such a homomorphism for the precosheaves in question.) It is easy to check that

$$
d D+D d=1
$$

as was to be shown.

CoRollary 4.3. If $\mathfrak{U}$ is a flabby cosheaf then for any covering $\mathfrak{U}$ of $X$ we have $\check{\mathfrak{F}}_{n}(\mathfrak{U} ; \mathfrak{H})=0$ for $n>0$. Consequently, $\breve{\mathfrak{L}}_{n}(X ; \mathfrak{A})=0$ for $n>0$.

Proof. This is a direct consequence of (2.3), (4.1) and (4.2).

THEOREM 4.4. Let $X$ be paracompact and let $\mathfrak{A}$ be a locally zero precosheaf on $X$. Then, for any covering $\mathfrak{H}$ of $X$, there is a refinement $\mathfrak{B}$ and a refinement projection $\mathfrak{B} \rightarrow \mathfrak{U}$ such that the induced $\operatorname{map} \check{C}_{n}(\mathfrak{B} ; \mathfrak{U}) \rightarrow \check{C}_{n}(\mathfrak{U} ; \mathfrak{U})$ is zero for all $n \geqq 0$.

Proof. We may assume that $\mathfrak{U}$ is locally finite and "self-indexing".

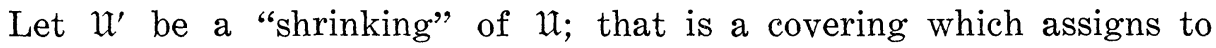
each $U \in \mathfrak{U}$ an open set $U^{\prime}$ with $\bar{U}^{\prime} \subset U$. For each $U \in \mathfrak{U}$ and $x \in U^{\prime}$ we choose an open set $W=W(x, U) \subset U^{\prime}$ with the property that whenever $U_{1}, \cdots, U_{n}$ are in $\mathfrak{U}$ and $W \cap U_{1}^{\prime} \cap \cdots \cap U_{n}^{\prime} \neq \varnothing$ then $W \subset U_{1} \cap \cdots \cap U_{n}$ and the map $\mathfrak{A}(W) \rightarrow \mathfrak{A}\left(U_{1} \cap \cdots \cap U_{n}\right)$ is trivial. The existence of such sets follows easily from local finiteness of $\mathfrak{U}$ and local triviality of $\mathfrak{A}$. We choose the refinement projection $W(x, U) \mapsto U$.

We claim that this covering satisfies the conclusion of the theorem. In fact, suppose that

$$
W_{i}=W\left(x_{i}, U_{i}\right), i=0, \cdots, n,
$$


and that $W_{0} \cap \cdots \cap W_{n} \neq \varnothing$. Then since

$$
W_{0} \cap \cdots \cap W_{n} \subset U_{0}^{\prime} \cap \cdots \cap U_{n}^{\prime}
$$

we must have $W_{i} \subset U_{0} \cap \cdots \cap U_{n}$ for each $i=0, \cdots, n$ and that $\mathfrak{U}\left(W_{i}\right) \rightarrow \mathfrak{A}\left(U_{0} \cap \cdots \cap U_{n}\right)$ is zero. Since

$$
\mathfrak{U}\left(W_{0} \cap \cdots \cap W_{n}\right) \longrightarrow \mathfrak{X}\left(U_{0} \cap \cdots \cap U_{n}\right)
$$

factors through $\mathfrak{A}\left(W_{0}\right)$, it is zero, and this finishes the proof.

CoROLlary 4.5. If $X$ is paracompact and if $\mathfrak{U}$ is a locally zero precosheaf on $X$ then $\breve{H}_{n}(X ; \mathfrak{U})=0$ for all $n \geqq 0$.

Corollary 4.6. Let $X$ be paracompact and let $h: \mathfrak{A} \rightarrow \mathfrak{B}$ be a local isomorphism. Then $h_{*}: \check{H}_{*}(X ; \mathfrak{A}) \rightarrow \check{H}_{*}(X ; \mathfrak{B})$ is an isomorphism.

Proof. Clearly this reduces to the two cases in which Coker $h=0$ or $\operatorname{Ker} h=0$. These cases are similar and we shall confine our attention to the first. Thus let $0 \rightarrow \Re \rightarrow \mathfrak{U} \rightarrow \mathfrak{B} \rightarrow 0$ be exact with $\Re$ locally zero. For each covering $\mathfrak{U}$ of $X$ we have the exact homology sequence

$$
\cdots \longrightarrow \check{H}_{n}(\mathfrak{U} ; \mathfrak{R}) \longrightarrow \check{H}_{n}(\mathfrak{U} ; \mathfrak{A}) \longrightarrow \check{H}_{n}(\mathfrak{U} ; \mathfrak{B}) \longrightarrow \check{H}_{n-1}(\mathfrak{U} ; \mathfrak{R}) \longrightarrow \cdots .
$$

Using (4.4) and the following lemma we see that the induced map $\check{H}_{n}(X ; \mathfrak{U}) \rightarrow \check{H}_{n}(X ; \mathfrak{B})$ is an isomorphism.

LEMMA 4.7. Let $\left\{A_{\alpha}, f_{\alpha, \beta}\right\},\left\{B_{\alpha}, g_{\alpha, \beta}\right\},\left\{C_{\alpha}, h_{\alpha, \beta}\right\}$ and $\left\{D_{\alpha}, k_{\alpha, \beta}\right\}$ be inverse systems of abelian groups and let

$$
A_{\alpha} \stackrel{\lambda_{\alpha}}{\longrightarrow} B_{\alpha} \stackrel{\mu_{\alpha}}{\longrightarrow} C_{\alpha} \stackrel{\nu \alpha}{\longrightarrow} D_{\alpha}
$$

be exact sequences commuting with projections. Assume that for each $\alpha$ there is a $\beta>\alpha$ such that $f_{\alpha, \beta}: A_{\beta} \rightarrow A_{\alpha}$ and $k_{\alpha, \beta}: D_{\beta} \rightarrow D_{\alpha}$ are zero. Then the induced map

$$
\mu: \lim _{\alpha} B_{\alpha} \longleftrightarrow \lim C_{\alpha}
$$

is an isomorphism.

Proof. Let $\left\{b_{\alpha}\right\}$ be the coordinates of an element of $\lim B_{\alpha}$ which is in the kernel of $\mu$. Then $\mu_{\alpha}\left(b_{\alpha}\right)=0$ for all $\alpha$ and thus $b_{\alpha}=\lambda_{\alpha}\left(a_{\alpha}\right)$ for some $a_{\alpha} \in A_{\alpha}$. By hypothesis there is a $\beta>\alpha$ such that $f_{\alpha \beta}=$ $0=k_{\alpha \beta}$. Thus $b_{\alpha}=g_{\alpha \beta}\left(b_{\beta}\right)=g_{\alpha \beta}\left(\lambda_{\beta}\left(a_{\beta}\right)\right)=\lambda_{\alpha}\left(f_{\alpha \beta}\left(a_{\beta}\right)\right)=0$ which shows that $\mu$ is monomorphic.

Now let $\left\{c_{\alpha}\right\}$ be a point in $\lim C_{\alpha}$. With $\beta$ as above we have 
$\nu_{\alpha}\left(c_{\alpha}\right)=\nu_{\alpha}\left(h_{\alpha \beta}\left(c_{\beta}\right)\right)=k_{\alpha \beta}\left(\nu_{\beta}\left(c_{\beta}\right)\right)=0$ so that $c_{\alpha}=\mu_{\alpha}\left(b_{\alpha}^{\prime}\right)$ for some $b_{\alpha}^{\prime} \in B_{\alpha}$. Let $\beta$ be as above (in relation to the given $\alpha$ ) and let $\gamma>\beta$ be arbitrary. Note that $b_{\beta}^{\prime} \equiv g_{\beta \gamma}\left(b_{\gamma}^{\prime}\right)$ (modulo $\operatorname{Im} \lambda_{\beta}$ ) since $\mu_{\beta}$ takes them to the same thing. Applying $g_{\alpha \beta}$ we obtain $g_{\alpha \beta}\left(b_{\beta}^{\prime}\right)=g_{\alpha \gamma}\left(b_{\gamma}^{\prime}\right)$ since $g_{\alpha \beta} \lambda_{\beta}=\lambda_{\alpha} f_{\alpha \beta}=0$. Let $b_{\alpha}$ be this common element $g_{\alpha \beta}\left(b_{\beta}^{\prime}\right)$ for $\beta>\alpha$ large. Clearly we have $g_{\gamma, \delta}\left(b_{\delta}\right)=b_{\gamma}$ for any $\delta>\gamma$ so that $\left\{b_{\alpha}\right\}$ is in $\lim B_{\alpha}$. Also $\mu_{\alpha}\left(b_{\alpha}\right)=\mu_{\alpha}\left(g_{\alpha \beta}\left(b_{\beta}^{\prime}\right)\right)=h_{\alpha \beta}\left(\mu_{\beta}\left(b_{\beta}^{\prime}\right)\right)=h_{\alpha \beta}\left(c_{\beta}\right)=c_{\alpha}$ which shows $\overleftarrow{\text { that }} \mu$ is epimorphic and completes the proof.

5. The reflector. For a precosheaf $\mathfrak{A}$ on $X$ we define the precosheaf $\Phi(\mathfrak{R})$ by

$$
\Phi(\mathfrak{U})=\breve{\mathfrak{L}}_{0}(X ; \widetilde{\mathfrak{U}})
$$

where $\tilde{\mathfrak{A}}$ is the maximal epiprecosheaf in $\mathfrak{A}$ of (1.5). Clearly $\Phi$ is a functor. There is a natural homomorphism

$$
\varphi(\mathfrak{U}): \Phi(\mathfrak{H}) \longrightarrow \mathfrak{A}
$$

given by the composition

$$
\breve{\mathfrak{S}}_{0}(X ; \widetilde{\mathfrak{U}}) \stackrel{\varepsilon *}{\longrightarrow} \widetilde{\mathfrak{U}} \longrightarrow \mathfrak{U} \text {. }
$$

Clearly $\varphi(\mathfrak{H})$ is an isomorphism when $\mathfrak{A}$ is a cosheaf.

If $h: \mathfrak{A} \rightarrow \mathfrak{B}$ is a homomorphism then we have the induced homomorphism $\Phi(h): \Phi(\mathfrak{U}) \rightarrow \Phi(\mathfrak{B})$. The following is a basic result:

THEOREM 5.1. If $h: \mathfrak{U} \rightarrow \mathfrak{B}$ is a local isomorphism of procosheaves and if either $\mathfrak{A}$ or $\mathfrak{B}$ is a cosheaf then $\Phi(h)$ is an isomorphism of $\Phi(\mathfrak{U})$ onto $\Phi(\mathfrak{B})$.

Proof. First assume that $\mathfrak{A}$ is a cosheaf. Note that $\Phi(\widetilde{\mathfrak{B}})=\Phi(\mathfrak{B})$. Also $h(\mathfrak{A}) \subset \widetilde{\mathfrak{B}}$ since $h(\mathfrak{A})$ is an epiprecosheaf. Clearly $h: \mathfrak{A} \rightarrow \widetilde{\mathfrak{B}}$ is also a local isomorphism. Thus we may assume that $\mathfrak{B}=\widetilde{\mathfrak{B}}$, that is, that $\mathfrak{B}$ is an epiprecosheaf. Coker $h$ is then also an epiprecosheaf and, being locally zero, it is zero. Let $\Re=\operatorname{Ker} h$.

Let $\mathfrak{U}=\left\{U_{i}\right\}$ be a covering of an open set $U \subset X$ so fine that $\Re\left(U_{i}\right) \rightarrow \Re(U)$ is zero for all $U_{i} \in \mathfrak{U}$. Consider the commutative diagram

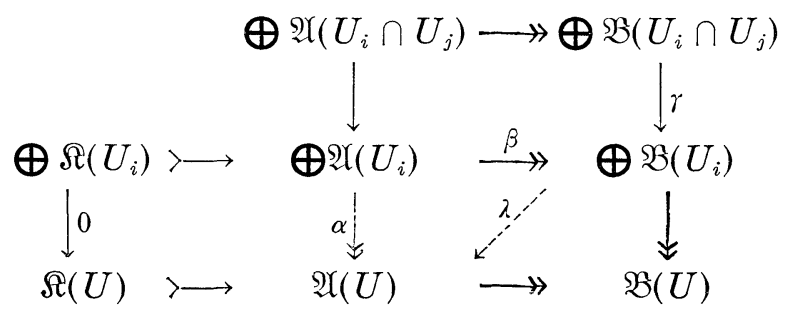


which is exact except for the right hand column. We see that $\operatorname{Ker} \alpha \supset \operatorname{Ker} \beta$ so that the additive relation $\lambda=\alpha \beta^{-1}$ is single valued and hence is a homomorphism. Clearly $\lambda$ is onto and diagram chasing shows that $\operatorname{Ker} \lambda=\beta(\operatorname{Ker} \alpha)=\operatorname{Im} \gamma$. Thus we have the induced isomorphism

$$
\Lambda: \check{H}_{0}(\mathfrak{U} ; \mathfrak{B}) \stackrel{\approx}{\longrightarrow} \mathfrak{U}(U) \approx \breve{H}_{0}(\mathfrak{M} ; \mathfrak{N}) .
$$

It is clear that $A$ is an inverse of the natural map

$$
h_{*}(\mathfrak{U}): \check{H}_{0}(\mathfrak{U} ; \mathfrak{U}) \longrightarrow \check{H}_{0}(\mathfrak{U} ; \mathfrak{B}) \text {. }
$$

Thus $h_{*}(\mathfrak{U})$ is an isomorphism and, upon passage to the limit over $\mathfrak{U}$, we see that $\Phi(h)(U)=\lim h_{*}(\mathfrak{U})$ is an isomorphism, as was to be shown.

Now suppose that $\mathfrak{B}$ is a cosheaf and that $\mathfrak{A}$ is arbitrary. Again Coker $h$ is an epiprecosheaf and hence it is zero. Let $\Re=\operatorname{Ker} h$ as before. Fix an open set $U \subset X$ for the time being and choose a covering $\left\{U_{i}\right\}$ of $U$ such that each $\Re\left(U_{i}\right) \rightarrow \Re(U)$ is trivial. Let $A=\operatorname{Im}\left\{\bigoplus \mathfrak{U}\left(U_{i}\right) \rightarrow \mathfrak{U}(U)\right\}$. Now the maps $\bigoplus \mathfrak{A}\left(U_{i}\right) \rightarrow \bigoplus \mathfrak{B}\left(U_{i}\right) \rightarrow \mathfrak{B}(U)$ are onto so that $A \rightarrow \mathfrak{B}(U)$ is onto. Consider the diagram

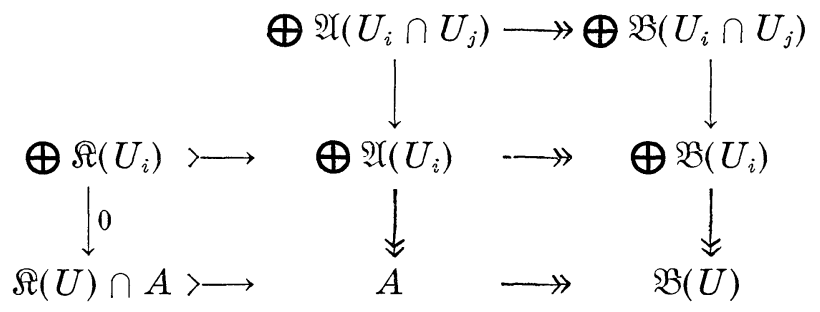

in which the rows and the right hand column are exact. Diagram chasing reveals that the left hand vertical map is onto and hence that $\mathscr{\Omega}(U) \cap A=0$. Thus the map $A \rightarrow \mathfrak{B}(U)$ is an isomorphism. It follows that a refinement of $\left\{U_{i}\right\}$ yields the same subgroup $A$ of $\mathfrak{A}(U)$. Thus in fact, in the notation of the proof of (1.5), $A=\mathfrak{A}_{1}(U)$ which is the set of elements of $\mathfrak{A}(U)$ in the image of $\bigoplus \mathfrak{A}\left(U_{i}\right) \rightarrow \mathfrak{A}(U)$ for every covering $\left\{U_{i}\right\}$ of $U$. This shows that $\mathfrak{A}_{1} \rightarrow \mathfrak{B}$ is an isomorphism and, since $\mathfrak{B}$ is a cosheaf, $\mathfrak{N}_{1}=\tilde{\mathfrak{U}}$ is a cosheaf. Thus

$$
h: \Phi(\mathfrak{U})=\widetilde{\mathfrak{U}} \stackrel{\approx}{\longrightarrow} \mathfrak{B}=\Phi(\mathfrak{B}) .
$$

The proof of the latter part of theorem shows more:

THEOREM 5.2. Let $h: \mathfrak{U} \rightarrow \mathfrak{B}$ be a local isomorphism and suppose that $\mathfrak{B}$ is a cosheaf. Then there exists a local isomorphism $k: \mathfrak{B} \rightarrow$ $\mathfrak{A}$ such that $h k=1$. 
Proof. $k$ is merely the inverse of the restriction $\widetilde{\mathfrak{A}} \rightarrow \mathfrak{B}$ of $h$ (followed, of course, by the inclusion $\tilde{\mathfrak{A}} \rightarrow \mathfrak{U}$ ). We have the split exact sequence

$$
0 \longrightarrow \Re \longrightarrow \mathfrak{A} \underset{k}{\stackrel{h}{\leftrightarrows}} \mathfrak{R} \longrightarrow 0
$$

and it follows that $\operatorname{Ker} k=0$ and that Coker $k \approx \operatorname{Ker} h=\Re$ is locally zero, so that $k$ is indeed a local isomorphism.

COROLlaRY 5.3. If a precosheaf $\mathfrak{A}$ is equivalent to a cosheaf $\mathfrak{B}$ then there is a local isomorphism $\mathfrak{B} \rightarrow \mathfrak{U}$.

COROLLARY 5.4. If the precosheaf $\mathfrak{i}$ is equivalent to a cosheaf (i.e. if it is smooth) then $\Phi(\mathfrak{U})$ is a cosheaf and $\varphi(\mathfrak{H}): \Phi(\mathfrak{U}) \rightarrow \mathfrak{U}$ is a local isomorphism.

Proof. By (5.3) there is a local isomorphism $h: \mathfrak{B} \rightarrow \mathfrak{U}$ for some cosheaf $\mathfrak{B}$. We have the diagram

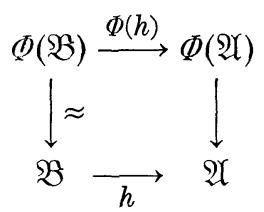

By (5.1) $\Phi(h)$ is an isomorphism and hence $\Phi(\mathfrak{H}) \rightarrow \mathfrak{U}$ may be identified with $h: \mathfrak{B} \rightarrow \mathfrak{U}$ and the result follows.

Corollary 5.5. Let $\mathscr{C}$ be the category of cosheaves on $X$ and $\mathscr{S}$ the category of smooth precosheaves (3.7). Then $\Phi$ is a reflector [9] from $\mathscr{S}$ to $\mathscr{C}$.

COROLlaRY 5.6. If $h: \mathfrak{U} \rightarrow \mathfrak{B}$ is a local isomorphism where $\mathfrak{A}, \mathfrak{B}$ are smooth then $\Phi(h): \Phi(\mathfrak{U}) \approx \Phi(\mathfrak{B})$.

Proof. By (5.3) there is a cosheaf $\mathbb{C}$ and a local isomorphism $k: \mathfrak{S} \rightarrow \mathfrak{A}$. The diagram

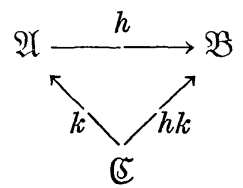

consists of local isomorphisms. In the induced diagram 


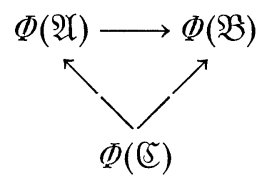

the diagonals are isomorphisms by (5.1) and hence $\Phi(h)$ is also an isomorphism.

CoROLlaRY 5.7. If $\mathfrak{A}$ and $\mathfrak{B}$ are cosheaves which are equivalent then they are isomorphic.

Proposition 5.8. If $\mathfrak{Y}^{\prime} \rightarrow \mathfrak{U} \rightarrow \mathfrak{U}^{\prime \prime}$ is a locally exact sequence of smooth precosheaves, then $\Phi\left(\mathfrak{U} \mathfrak{U}^{\prime}\right) \rightarrow \Phi(\mathfrak{U}) \rightarrow \Phi\left(\mathfrak{U}^{\prime \prime}\right)$ is also locally exact.

Proof. Apply (3.9) and (5.4) to the diagram

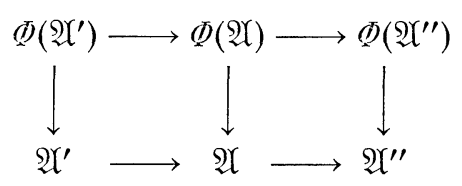

REMARK 5.9. Not every precosheaf is smooth. For a simple example let $X$ be the unit interval and let $\mathfrak{A}$ be the precosheaf on $X$ which assigns to $U$ the group of singular 1-chains of $U$. The associated epiprecosheaf $\tilde{\mathfrak{A}}$ clearly has $\tilde{\mathfrak{A}}(U)$ equal to the subgroup generated by the constant singular 1-simplices. The inclusion $\widetilde{\mathfrak{A}} \rightarrow \mathfrak{A}$ is clearly not a local isomorphism and it follows that $\Phi(\mathfrak{U}) \rightarrow \mathfrak{A}$ cannot be a local isomorphism. This would contradict (5.4) if $\mathfrak{U}$ were smooth.

REMARK 5.10. If $\mathfrak{A}$ is a smooth precosheaf then the inclusion $\widetilde{\mathfrak{U}} \rightarrow \mathfrak{U}$ is a local isomorphism. Thus, if $X$ is completely paracompact, then it follows from (4.6) that the induced map $\Phi(\mathfrak{A})=\check{\mathfrak{E}}_{0}(X ; \widetilde{\mathfrak{U}}) \rightarrow$ $\breve{\mathfrak{g}}_{0}(X ; \mathfrak{A})$ is an isomorphism. This probably does not hold without the paracompactness assumption. We also remark that the (exact) analogue of $\Phi$ in the theory of eshaves is the coreflector assigning to any presheaf its associated sheaf, and similarly the analogue of $\breve{\mathfrak{K}}_{0}(X ; \mathfrak{A})$ is the "completion" of a presheaf in Spanier's terminology [10, p. 325].

REMARK 5.11. Constant precosheaves on locally connected spaces are smooth. For this see the first part of $\S 9$ in which the local compactness condition is unnecessary. The associated cosheaves are called constant cosheaves.

6. Spectral sequences. As usual a differential cosheaf is a 
sequence $\left\{\mathfrak{A}_{i}\right\}$ of cosheaves together with a "differential" $d: \mathfrak{U}_{i} \rightarrow \mathfrak{A}_{i-1}$ with $d^{2}=0$.

Let $\mathfrak{A}_{*}$ be a flabby differential cosheaf which is bounded below (i.e. $\mathfrak{A}_{i}=0$ for $i<i_{0}$ some $i_{0}$ ). Given a covering $\mathfrak{U}$ of $X$ consider the double complex

$$
L_{p, q}=\check{C}_{p}\left(\mathfrak{M} ; \mathfrak{N}_{q}\right) \text {. }
$$

There are two spectral sequences of this double complex. In one of them we have the $E_{p, q}^{1}$-term

$$
{ }^{\prime} H_{q}\left(L_{*, p}\right)=\check{H}_{q}\left(\mathfrak{U} ; \mathfrak{A}_{p}\right)=\left\{\begin{array}{cc}
\mathfrak{A}_{p}(X) & \text { for } q=0 \\
0 & \text { for } q \neq 0
\end{array}\right.
$$

by (4.3). Thus the $E_{p, q}^{2}$-term is

$$
{ }^{\prime} H_{p}^{\prime} H_{q}\left(L_{*, *}\right)= \begin{cases}H_{p}\left(\mathfrak{H}_{*}(X)\right) & \text { for } q=0 \\ 0 & \text { for } q \neq 0 .\end{cases}
$$

Since this spectral sequence degenerates we have the natural isomorphism

$$
H_{n}\left(L_{*}\right) \approx H_{n}\left(\mathfrak{A}_{*}(X)\right)
$$

where $L_{*}$ is the total complex of $L_{*, *}$.

In the other spectral sequence we have

$$
E_{p, q}^{1}=" H_{q}\left(L_{p, *}\right)=\check{C}_{p}\left(\mathfrak{U} ; \mathfrak{E}_{q}\left(\mathfrak{U}_{*}\right)\right)
$$

and hence

$$
E_{p, q}^{2}=\check{H}_{p}\left(\mathfrak{U} ; \mathfrak{S}_{q}\left(\mathfrak{H}_{*}\right)\right) .
$$

By the assumption that $\mathfrak{U}_{*}$ is bounded below this spectral converges to $H_{*}\left(L_{*}\right)$. Thus we have a spectral sequence $E_{p, q}^{r}(\mathfrak{U})$ with

$$
E_{p, q}^{2}(\mathfrak{U})=\check{H}_{p}\left(\mathfrak{U} ; \mathfrak{S}_{q}\left(\mathfrak{A}_{*}\right)\right) \Longrightarrow H_{p+q}\left(\mathfrak{A}_{*}(X)\right) \text {. }
$$

These spectral sequences are clearly functorial in the coverings $\mathfrak{U}$ as well as in $\mathfrak{U}_{*}$.

7. Coresolutions. By an $N$-coresolution of a cosheaf $\mathfrak{A}$ we mean a differential cosheaf $\mathfrak{U}_{*}$ together with an augmentation $\varepsilon: \mathfrak{U}_{0} \rightarrow \mathfrak{A}$ such that the sequence

$$
\mathfrak{A}_{N+1} \longrightarrow \cdots \longrightarrow \mathfrak{A}_{2} \stackrel{d}{\longrightarrow} \mathfrak{A}_{1} \stackrel{d}{\longrightarrow} \mathfrak{A}_{0} \stackrel{\varepsilon}{\longrightarrow} \mathfrak{A} \longrightarrow 0
$$

is locally exact. Note that, by (2.1), the portion $\mathfrak{A}_{1} \rightarrow \mathfrak{A}_{0} \rightarrow \mathfrak{A} \rightarrow 0$ is actually exact. IWe remark that everything we do remains valid if 
we replace the statement " $N$-coresolution on $X$ " by " $(N-1)$-coresolution and $H_{N}\left(\mathfrak{A}_{*}(U)\right) \rightarrow H_{N}\left(\mathfrak{A}_{*}(X)\right)$ is trivial for some basis of open sets $U \subset X$ "; see the remark (10.5).]

In this section we will fix an $N \geqq 0$ and assume that $\mathfrak{A}_{*}$ is a flabby $N$-coresolution of a given cosheaf $\mathfrak{A}$ and shall study the spectral sequences (6.1). We also assume that $X$ is paracompact.

Lemma 7.1. If $\mathfrak{U}$ is sufficiently fine then the natural projection $H_{n}\left(\mathfrak{U}_{*}(X)\right) \rightarrow E_{n, 0}^{\infty}(\mathfrak{U})$ is an isomorphism for all $n \leqq N$.

Proof. Given any covering $\mathfrak{U}$ we can find by (4.4) a refinement $\mathfrak{u}^{\prime}$ such that $E_{p, q}^{2}\left(\mathfrak{u}^{\prime}\right) \rightarrow E_{p, q}^{2}(\mathfrak{u})$ is zero for all $p, q$ with $q \neq 0$ and $p+q \leqq N$. It follows that $E_{p, q}^{r}\left(\mathfrak{U}^{\prime}\right) \rightarrow E_{p, q}^{r}(\mathfrak{u})$ is zero for all $r \geqq 2$.

Now recall that $H_{n}\left(\mathfrak{U}_{*}(X)\right)$ is filtered by submodules

$$
H_{n}\left(\mathfrak{H}_{*}(X)\right)=J_{n} \supset J_{n-1} \supset \cdots \supset J_{0} \supset 0
$$

such that $E_{p, n-p}^{\infty}(\mathfrak{U}) \approx J_{p} / J_{p-1}$. Similarly $H_{n}\left(\mathfrak{U}_{*}(X)\right)$ is filtered by $J_{p}^{\prime}$ with $J_{p}^{\prime} / J_{p-1}^{\prime} \approx E_{p, n-p}^{\infty}\left(\mathfrak{u}^{\prime}\right)$. Since the refinement has no effect on $H_{n}\left(\mathfrak{A}_{*}(X)\right)$ we see that it induces an isomorphism $J_{n}^{\prime} \rightarrow J_{n}$ and that for $p<n, J_{p}^{\prime} \rightarrow J_{p}$ has image in $J_{p-1}$ (and, of course, is a monomorphism). It follows that $J_{0}^{\prime}=0$. A similar argument on a similar refinement $\mathfrak{U}^{\prime \prime}$ of $\mathfrak{U}^{\prime}$ will show that $J_{1}^{\prime \prime}=0$, etc. Thus $\mathfrak{u}$ may be assumed to be so fine that $J_{p}=0$ for all $p<n$. This implies the desired result.

Returning to the general discussion, let $\mathfrak{u}=\mathfrak{U}_{0}$ be as in (7.1) for some fixed $n \leqq N$. Construct refinements $\mathfrak{u}_{i}$ of $\mathfrak{u}_{i-1}$ for $i=1,2, \cdots, n$ such that

$$
E_{p q}^{2}\left(\mathfrak{U}_{i}\right) \longrightarrow E_{p, q}^{2}\left(\mathfrak{U}_{i-1}\right)
$$

is trivial for all $p, q$ with $p+q \leqq n$ and $q \neq 0$.

We see that the image of $E_{n, 0}^{2}\left(\mathfrak{U}_{n}\right) \rightarrow E_{n, 0}^{2}\left(\mathfrak{U}_{n-1}\right)$ consists of $d_{2}$-cycles and hence induces a homomorphism

$$
E_{n, 0}^{2}\left(\mathfrak{U}_{n}\right) \longrightarrow E_{n, 0}^{3}\left(\mathfrak{U}_{n-1}\right)
$$

similarly we obtain homomorphisms

$$
E_{n, 0}^{3}\left(\mathfrak{U}_{n-1}\right) \longrightarrow E_{n, 0}^{4}\left(\mathfrak{U}_{n-2}\right) \longrightarrow \cdots \longrightarrow E_{n, 0}^{\infty}\left(\mathfrak{U}_{0}\right)
$$

This provides the commutative diagram 


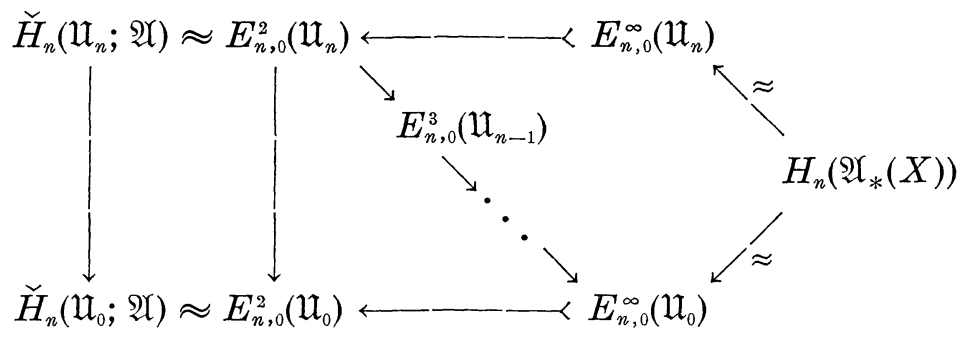

[We remark that this diagram exists for $n=N+1$, except that the right hand maps are then only epimorphisms.]

Letting $\mathfrak{U}_{n}=\mathfrak{u}^{\prime}$ this yields a diagram

$$
\check{H}_{n}\left(\left.\mathfrak{U}\right|^{\prime} ; \mathfrak{U} \mathfrak{U}^{\prime} ; \mathfrak{U}\right) H_{n} H_{n}\left(\mathfrak{U}_{*}(X)\right)
$$

where $j$ is the refinement projection, the $\lambda^{\prime}$ s are edge homomorphisms, and $\mu$ is induced by the diagonal composition in the last diagram. Checking the definitions we see easily that we have the following commutativity relations:

$$
\left\{\begin{aligned}
\lambda & =j \lambda^{\prime} \\
j & =\lambda \mu \\
\mu \lambda^{\prime} & =1 .
\end{aligned}\right.
$$

It follows immediately that $\lambda^{\prime}$ is a monomorphism and that

$$
\operatorname{Im} j=\operatorname{Im} \lambda \text {. }
$$

Clearly we may assume that $\mathfrak{U}$ is also so fine that $\lambda$ is a monomorphism and it follows that

$$
j: \operatorname{Im} \lambda^{\prime} \stackrel{\approx}{\longrightarrow} \operatorname{Im} \lambda .
$$

These considerations prove, in particular, the following result:

THeOREM 7.2. Let $X$ be paracompact and let $\mathfrak{A}_{*}$ be a flabby $N$-coresolution of a cosheaf $\mathfrak{X}$ on $X$. Then for $n \leqq N$ the edge homomorphisms $\lambda_{\mathfrak{n}}: H_{n}\left(\mathfrak{U}_{*}(X)\right) \rightarrow \check{H}_{n}(\mathfrak{U} ; \mathfrak{U})$ of the spectral sequences (6.1) induce an isomorphism in the limit over $\mathfrak{u}$ :

$$
\lambda_{X}: H_{n}\left(\mathfrak{A}_{*}(X)\right) \stackrel{\approx}{\longrightarrow} \breve{H}_{n}(X ; \mathfrak{⿰}) .
$$

In fact we have shown that if $\mathfrak{U}$ is a sufficiently fine covering of $X$, then $\lambda_{\mathfrak{u}}: H_{n}\left(\mathfrak{A}_{*}(X)\right) \rightarrow \check{H}_{n}(\mathfrak{U} ; \mathfrak{A})$ is a monomorphism onto the 
image of the canonical projection

$$
\pi: \check{H}_{n}(X ; \mathfrak{A}) \longrightarrow \check{H}_{n}(\mathfrak{U} ; \mathfrak{U})
$$

and, moreover, that $\pi$ is a monomorphism. Moreover, given this $\mathfrak{u}$, then for any sufficiently fine refinement $\mathfrak{u}^{\prime}$ of $\mathfrak{u}$ we have $\operatorname{Im} j=$ $\operatorname{Im} \lambda=\operatorname{Im} \pi$ and $j: \operatorname{Im} \lambda^{\prime} \stackrel{\approx}{\longrightarrow} \operatorname{Im} \lambda$. The notation is as in the discussion above (7.2).)

REMARK 7.3. It might be thought that, in (7.2), $\lambda$ is an epimorphism in degree $N+1$, but this is not generally true. An interesting counter-example, in the application to singular homology, is given by Mardešic in [8]. One can easily see, however, that for every covering $\mathfrak{u}$ of $X$ the images of $H_{N+1}\left(\mathfrak{H}_{*}(X)\right)$ and of $\check{H}_{N+1}(X ; \mathfrak{A})$ in $\check{H}_{N+1}(\mathfrak{U} ; \mathfrak{A})$ coincide.

8. Relative homology. To this point we have restricted our attention to absolute homology in order to maintain reasonably uncluttered notation. In this section we shall show how the results of the last two sections can be extended to the relative case.

Let $A \subset X$ be an arbitrary subspace. For a precosheaf $\mathfrak{U}$ on $A$ we define a precosheaf $\mathfrak{U}^{x}$ on $X$ by

$$
\mathfrak{H}^{X}(U)=\mathfrak{H}(U \cap A) .
$$

Note that $\mathfrak{X}^{X}$ is a cosheaf if and only if $\mathfrak{X}$ is and that it is flabby if and only if $\mathfrak{A}$ is.

A covering of the pair $(X, A)$ is a pair $\left(\mathfrak{u}, \mathfrak{u}_{0}\right)$ where $\mathfrak{U}$ is a covering of $X$ and $\mathfrak{u}_{0} \subset \mathfrak{U}$ covers $A$ in $X$. If $\mathfrak{U}$ is a precosheaf on $A$ then we have the obvious isomorphism

$$
\check{C}_{*}\left(\mathfrak{U}_{0} ; \mathfrak{U}^{X}\right) \approx \check{C}_{*}\left(\mathfrak{U}_{0} \cap A ; \mathfrak{U}\right)
$$

where $\mathfrak{U}_{0} \cap A=\left\{U \cap A \mid U \in \mathfrak{U}_{0}\right\}$.

Suppose that $\mathfrak{U}$ and $\mathfrak{B}$ are precosheaves on $A$ and $X$ respectively and that we are given a monomorphism of precosheaves

$$
\eta: \mathfrak{A}^{x} \succ \mathfrak{B} \text {. }
$$

We obtain the induced monomorphism

$$
\check{C}_{*}\left(\mathfrak{U}_{0} \cap A ; \mathfrak{U}\right)>\longrightarrow \check{C}_{*}\left(\mathfrak{U}_{0} ; \mathfrak{B}\right)
$$

and shall denote its cokernel by

$$
\check{C}_{*}\left(\mathfrak{U}, \mathfrak{U}_{0} ; \mathfrak{B}, \mathfrak{U}\right) \text {. }
$$

Thus we have the natural exact sequence 


$$
\check{C}_{*}\left(\mathfrak{U}_{0} \cap A ; \mathfrak{A}\right) 〉 \longrightarrow \check{C}_{*}(\mathfrak{U} ; \mathfrak{B}) \succ \check{C}_{*}\left(\mathfrak{U}, \mathfrak{U}_{0} ; \mathfrak{B}, \mathfrak{N}\right)
$$

of chain complexes. As usual we denote the homology of this chain complex by

$$
\check{H}_{*}\left(\mathfrak{U}, \mathfrak{U}_{0} ; \mathfrak{B}, \mathfrak{U}\right)
$$

and obtain from (8.2) the usual long homology sequence. The inverse limit over refinements of pairs of coverings yields, as usual, a group denoted by $\check{H}_{*}(X, A ; \mathfrak{B}, \mathfrak{U})$.

Now assume that $\mathfrak{X}$ and $\mathfrak{B}$ are flabby cosheaves. Then (4.3), applied to the homology sequence of (8.2), yields the conclusion that $\check{H}_{n}\left(\mathfrak{U}, \mathfrak{U}_{0} ; \mathfrak{B}, \mathfrak{U}\right)=0$ for $n>1$ and yields the exact sequence

$$
\begin{aligned}
0 \longrightarrow \check{H}_{1}\left(\mathfrak{U}, \mathfrak{u}_{0} ; \mathfrak{B}, \mathfrak{I}\right) \longrightarrow \check{H}_{0}\left(\mathfrak{U}_{0} \cap A ; \mathfrak{Y}\right) \\
\longrightarrow \check{H}_{0}(\mathfrak{U} ; \mathfrak{B}) \longrightarrow \check{H}_{0}\left(\mathfrak{U}, \mathfrak{U}_{0} ; \mathfrak{B}, \mathfrak{U}\right) \longrightarrow 0
\end{aligned}
$$

The two middle terms of this sequence are canonically isomorphic to $\mathfrak{U}^{X}$ and $\mathfrak{B}$ respectively with $\eta$ as the homomorphism between them. Thus, by assumption, we have

$$
H_{n}\left(\mathfrak{U}, \mathfrak{U}_{0} ; \mathfrak{B}, \mathfrak{U}\right) \approx \begin{cases}0 & \text { for } n \geqq 1 \\ \text { Coker } \eta: \mathfrak{I}^{x} \longrightarrow \mathfrak{B} & \text { for } n=0\end{cases}
$$

when $\mathfrak{A}$ and $\mathfrak{B}$ are flabby cosheaves.

Now suppose that $\mathfrak{U}_{*}$ and $\mathfrak{B}_{*}$ are flabby differential cosheaves on $A$ and $X$ respectively, which are bounded below. Also suppose that we are given an exact sequence

$$
0 \longrightarrow \mathfrak{H}_{*}^{X} \longrightarrow \mathfrak{B}_{*} \longrightarrow \mathfrak{C}_{*} \longrightarrow 0
$$

(defining $\mathfrak{c}_{*}$ ) of differential cosheaves.

Given the pair of coverings $\left(\mathfrak{U}, \mathfrak{U}_{0}\right)$ we consider the double complex

$$
L_{p, q}\left(\mathfrak{u}, \mathfrak{U}_{0}\right)=\check{C}_{p}\left(\mathfrak{U}, \mathfrak{U}_{n} ; \mathfrak{B}_{q}, \mathfrak{X}_{q}\right) .
$$

As in $\S 6$ it follows immediately from (8.3) that there is a natural spectral sequence $E_{p, q}^{r}\left(\mathfrak{u}, \mathfrak{u}_{0}\right)$ with

$$
E_{p, q}^{\lrcorner}\left(\mathfrak{H}, \mathfrak{H}_{0}\right)={ }^{\prime} H_{q}\left(L_{p, *}\left(\mathfrak{H}, \mathfrak{U}_{0}\right)\right) .
$$

and

$$
E_{p, q}^{2}\left(\mathfrak{u}, \mathfrak{u}_{0}\right)={ }^{\prime} H_{p}^{\prime \prime} H_{q}\left(L_{*, *}\left(\mathfrak{u}, \mathfrak{u}^{0}\right)\right) \Rightarrow H_{p+q}\left(\mathfrak{S}_{*}(X)\right) .
$$

Now we suppose that $\mathfrak{U}_{*}$ and $\mathfrak{B}_{*}$ are flabby $N$-coresolutions of cosheaves $\mathfrak{A}$ and $\mathfrak{B}$ on $A$ and $X$ respectively. (Note that, unless $A$ is closed, it does not generally follow that $\mathfrak{C}_{*}$ is a coresolution of 
Coker $\left(\mathfrak{U}^{x} \rightarrow \mathfrak{B}\right)$.)

Exact sequences of the form (8.2) yield, for the " $H$ homology, the exact sequence

$$
\begin{aligned}
& \cdots \longrightarrow \check{C}_{p}\left(\mathfrak{U}_{0} \cap A ; \mathfrak{S}_{q}\left(\mathfrak{A}_{*}\right)\right) \longrightarrow \check{C}_{p}\left(\mathfrak{U} ; \mathfrak{S}_{q}\left(\mathfrak{B}_{*}\right)\right) \longrightarrow E_{p, q}^{1}\left(\mathfrak{U}, \mathfrak{U}_{0}\right) \longrightarrow \\
& \cdots
\end{aligned}
$$

(here $\widetilde{\mathfrak{E}}_{0}\left(\mathfrak{U}_{*}\right)=\operatorname{Ker}\left\{\mathfrak{S}_{0}\left(\mathfrak{U}_{*}\right) \rightarrow \mathfrak{U}\right\}=0$ ). Moreover we have the exact sequence

$$
\check{C}_{p}\left(\mathfrak{U}_{0} \cap A ; \mathfrak{U}\right) \longrightarrow \check{C}_{p}(\mathfrak{U} ; \mathfrak{B}) \longrightarrow E_{p, 0}^{1}\left(\mathfrak{H}, \mathfrak{U}_{0}\right) \longrightarrow 0 \text {. }
$$

By (8.8) we have

$$
\left\{\begin{array}{l}
E_{p, 0}^{1}\left(\mathfrak{U}, \mathfrak{U}_{0}\right)=\check{C}_{p}\left(\mathfrak{U}, \mathfrak{U}_{0} ; \mathfrak{B}, \mathfrak{U}\right) \\
E_{p, 0}^{2}\left(\mathfrak{H}, \mathfrak{U}_{0}\right)=\check{H}_{p}\left(\mathfrak{U}, \mathfrak{U}_{0} ; \mathfrak{B}, \mathfrak{U}\right)
\end{array}\right.
$$

Moreover if $X$ and $A$ are both paracompact then, by (8.7) and (4.4) (twice), there exists a refining pair $\left(\mathfrak{N}, \mathfrak{B}_{0}\right)$ of $\left(\mathfrak{U}, \mathfrak{U}_{0}\right)$ and a refinement projection such that the induced homomorphism

$$
E_{p, q}^{1}\left(\mathfrak{B}, \mathfrak{B}_{0}\right) \longrightarrow E_{p, q}^{1}\left(\mathfrak{u}, \mathfrak{U}_{0}\right)
$$

is trivial for all $p$ and all $q \neq 0$ (with $p+q \leqq N$ ).

We now have all the information necessary to repeat the arguments in $\S 7$ word for word. The final result is the following extension of (7.2):

Theorem 8.10. Let $A \subset X$ both be paracompact and let $\mathfrak{U}_{*}$ and $\mathfrak{B}_{*}$ be flabby $N$-coresolutions of cosheaves $\mathfrak{U}$ and $\mathfrak{B}$ on $A$ and $X$ respectively. Suppose $0 \rightarrow \mathfrak{H}_{*}^{X} \rightarrow \mathfrak{B}_{*} \rightarrow \mathfrak{S}_{*} \rightarrow 0$ is an exact sequence of differential cosheaves. Then for $n \leqq N$ the edge homomorphisms $H_{n}\left(\mathfrak{S}_{*}(X)\right) \rightarrow E_{n, 0}^{2}\left(\mathfrak{U}, \mathfrak{U}_{0}\right)=\check{H}_{n}\left(\mathfrak{H}, \mathfrak{U}_{0} ; \mathfrak{B}, \mathfrak{A}\right)$ of the spectral sequences (8.6) induce an isomorphism in the limit over $\left(\mathfrak{u}, \mathfrak{u}_{0}\right)$ :

$$
H_{n}\left(\mathfrak{\complement}_{*}(X)\right) \stackrel{\approx}{\longrightarrow} \check{H}_{n}(X, A ; \mathfrak{B}, \mathfrak{A}) \text {. }
$$

We note that it is also easy to see that, via (8.10) and (7.2), the exact sequence

$$
\cdots \longrightarrow H_{n}\left(\mathfrak{U}_{*}(A)\right) \longrightarrow H_{n}\left(\mathfrak{B}_{*}(X)\right) \longrightarrow H_{n}\left(\mathfrak{\complement}_{*}(X)\right) \longrightarrow H_{n-1}\left(\mathfrak{A}_{*}(A)\right) \longrightarrow \cdots
$$

is identified with the sequence

$$
\cdots \longrightarrow \breve{H}_{n}(A ; \mathfrak{A}) \longrightarrow \check{H}_{n}(X ; \mathfrak{B}) \longrightarrow \check{H}_{n}(X, A ; \mathfrak{B}, \mathfrak{N}) \longrightarrow \check{H}_{n-1}(A, \mathfrak{N}) \longrightarrow \cdots
$$

which is the inverse limit of similar exact sequences of the appropriate Čech groups of coverings. In particular, it follows that this homology 
sequence is exact, a fact that clearly limits the possibilities of the existence of the hypothesized coresolutions. This question will be taken up in later sections.

By introducing compact carriers in the Čech theory we may replace the paracompactness condition in (8.10) by a local compactness condition. The idea for doing this is due to Mardešic [8]. It involves the observation that any compact set in a locally compact space is contained in an open relatively compact $F_{o}$ (and hence paracompact) set.

We define, for $(X, A)$ a locally compact pair,

$$
\check{H}_{n}^{c}(X, A ; \mathfrak{B}, \mathfrak{U})=\underline{\lim } \check{H}_{n}(U, V ; \mathfrak{B}, \mathfrak{U})
$$

where $U$ and $V \subset U \cap A$ range over the open, relatively compact, subsets of $X$ and $A$ respectively. By the above remark we may also assume that $U$ and $V$ are paracompact in this limit. By making the elementary observation that, for any cosheaf $\sqrt{5}, \sqrt{5}(X) \approx \lim \sqrt{ }(U), U$ relatively compact, [and hence $\left.H_{n}\left(\mathfrak{\Im}_{*}(X)\right) \approx \lim H_{n}\left(\mathfrak{S}_{*}(U)\right)\right]$ we obtain the following corollary of (8.10):

COROLlary 8.11. With the same hypotheses as (8.10) except that $(X, A)$ are assumed to be locally compact rather than paracompact, we obtain the canonical isomorphism

$$
H_{n}\left(\complement_{*}(X)\right) \approx \check{H}_{n}^{c}(X, A ; \mathfrak{B}, \mathfrak{R}) .
$$

We wish to generalize (4.6) to the relative case. Thus assume that $\mathfrak{A}_{1}, \mathfrak{U}_{2}$ are precosheaves on $A$ and $\mathfrak{B}_{1}, \mathfrak{B}_{2}$, on $X$ and assume that homomorphisms $\mathfrak{A}_{i}^{X} \succ \mathfrak{B}_{i}, h: \mathfrak{A}_{1} \rightarrow \mathfrak{A}_{2}$ and $k: \mathfrak{B}_{1} \rightarrow \mathfrak{B}_{2}$ are given such that

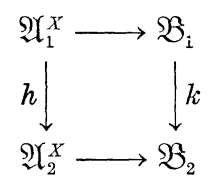

commutes. Then we have:

Proposition 8.12. If $(X, A)$ above is a paracompact (respectively, locally compact) pair and $h$ and $k$ above are local isomorphisms, then the induced map

$$
\check{H}_{*}\left(X, A ; \mathfrak{B}_{1}, \mathfrak{A}_{1}\right) \longrightarrow \check{H}_{*}\left(X, A ; \mathfrak{B}_{2}, \mathfrak{A}_{2}\right)
$$

is an isomorphism (respectively, the same for the groups $\check{H}_{*}^{c}$ ).

The proof is essentially the same as that of (4.6) and will not be repeated. 
9. Borel-Moore homology. In this section we confine our attention to locally compact spaces and take the base ring $L$ to be a principal ideal domain. We use the notation $H_{*}^{c}(X ; \mathscr{A})$ for the Borel-Moore homology with compact supports and with coefficients in the sheaf $\mathscr{A}$; see [1, Chap. V].

The constant cosheaf $\mathcal{Z}$ is the precosheaf assigning to $U$ the free $L$-module on the components of $U$ and for an $L$-module $M$ the associated constant cosheaf $\mathfrak{M}$ is defined by $\mathfrak{M}(U)=\mathfrak{L}(U) \otimes M$. Summation yields a homomorphism $\mathfrak{M} \rightarrow M$ of precosheaves (where $M(U)=M$ ) and it is clear that this is a local isomorphism if $X$ is locally connected. Thus $\Phi(M) \approx \mathfrak{M}$ when $X$ is locally connected.

We wish to associate with every sheaf $\mathscr{A}$ on a locally connected space $X$ a certain associated cosheaf on $X$.

Proposition 9.1. Let $X$ be locally connected (and locally compact). Then for any sheaf $\mathscr{A}$ on $X$, the precosheaf $H_{0}^{c}(\cdot ; \mathscr{A})$ is a cosheaf. If $X$ is $\operatorname{clc}_{L}^{1}$, or if $L$ is a field, and if $\mathscr{A}=M$ is constant then this cosheaf is the constant cosheaf $\mathfrak{M}=\Phi(M)$.

Proof. Let $\mathscr{L}_{p}=\mathscr{C}_{p}(X ; L)$ be the sheaf of germs of Borel-Moore $p$-chains as defined in $\left[1\right.$, p. 184], and let $\mathscr{K}=\operatorname{Ker}\left\{d: \mathscr{L}_{0} \rightarrow \mathscr{L}_{-1}\right\}$. The proof of Theorem 5.12, p. 201 of [1] shows that $\mathscr{K} \otimes \mathscr{A}$ is $c$-soft and that the sequence

$$
\Gamma_{c}(\mathscr{K} \otimes \mathscr{A} \mid U)>\longrightarrow \Gamma_{c}\left(\mathscr{L}_{0} \otimes \mathscr{A} \mid U\right) \longrightarrow \Gamma_{c}\left(\mathscr{L}_{-1} \otimes \mathscr{A} \mid U\right)
$$

is exact, so that the first term consists of the zero-cycles of $U$. Thus we have, by definition, the exact sequence

$$
\Gamma_{c}\left(\mathscr{L}_{1} \otimes \mathscr{A} \mid U\right) \longrightarrow \Gamma_{c}(\mathscr{K} \otimes \mathscr{A} \mid U) \longrightarrow H_{0}^{c}(U ; \mathscr{A}) \longrightarrow 0 .
$$

Since the first two terms are cosheaves [1, p. 176] the last is also a cosheaf by (1.1). The last statement follows immediately from $(\mathrm{V}, 5.11)$ and $(\mathrm{V}, 3.10)$ of $[1]$.

This proposition is used only in case $\mathscr{A}$ is locally constant and, then, merely to prove the existence of a related "locally constant" cosheaf.

From the proof of (9.1) we see that, when $X$ is locally connected, the canonical Borel-Moore chain complex $C_{*}^{c}(X ; \mathscr{A})$ [i.e. $\left.\Gamma_{c}\left(\mathscr{C}_{*}(X ; L) \otimes \mathscr{S}^{\prime}\right)\right]$ may be replaced by a chain complex vanishing in negative degrees, without altering the homology. That is we define

$$
\bar{C}_{p}^{c}(U ; \mathscr{A})=\left\{\begin{array}{lr}
C_{p}^{c}(U ; \mathscr{A}) & \text { if } p>0 \\
\operatorname{Ker}\left\{C_{0}^{c}(U ; \mathscr{A}) \longrightarrow C_{-1}^{c}(U ; \mathscr{A})\right\} & \text { if } p=0 \\
0 & \text { if } p<0
\end{array}\right.
$$


and note that, by the proof of (9.1), the precosheaf

$$
\bar{\Im}_{p}^{c}(X ; \mathscr{A}): U \longmapsto \bar{C}_{p}^{c}(U ; \mathscr{A})
$$

is a flabby cosheaf.

Now suppose $A \subset X$ is also locally connected. By general results in $[1, V .5]$ we have an exact sequence

$$
0 \longrightarrow \overline{\mathfrak{S}}_{p}^{c}(A ; \mathscr{A} \mid A)^{X} \longrightarrow \overline{\mathfrak{S}}_{p}^{c}(X ; \mathscr{A}) \longrightarrow \overline{\mathfrak{S}}_{p}^{c}(X, A ; \mathscr{A}) \longrightarrow 0
$$

(which defines the right hand term).

Proposition 9.2. If $X$ is $h l c_{L}^{N}$ and if $\mathscr{A}$ is locally constant, then $\bar{\complement}_{*}^{c}(X ; \mathscr{A})$ is a flabby $N$-coresolution of the cosheaf $H_{0}^{c}(\cdot ; \mathscr{A})$.

Proof. It is required to show that the precosheaf

$$
U \longmapsto H_{n}^{c}(U ; \mathscr{A})
$$

is locally zero for $0<n \leqq N$. For $\mathscr{A}=L$ this is true by definition $[1$, p. 253]. For $\mathscr{A}=M$, any constant sheaf, the result follows from the universal coefficient formula [1, p. 188]. The locally constant case follows immediately.

Now from (9.2), (8.10), and (8.11) we immediately obtain the following result:

TheOREm 9.3. Let $A \subset X$ both be locally compact and $h l c_{L}^{v}$. Let $\mathscr{A}$ be a locally constant sheaf on $X$. Then for $n \leqq N$ we have a canonical isomorphism

$$
H_{n}^{c}(X, A ; \mathscr{A}) \approx \check{H}_{n}^{c}\left(X, A ; H_{0}^{c}(\cdot ; \mathscr{A})\right) .
$$

If $X$ and $A$ are also paracompact, then these are isomorphic to $\check{H}_{n}\left(X, A ; H_{0}^{c}(\cdot ; \mathscr{A})\right)$.

[Technically the coefficients on the right should be the pair $\left.H_{0}^{c}(\cdot ; \mathscr{A}), H_{0}^{c}(\cdot ; \mathscr{A} \mid A).\right]$

Corollary 9.4. Let $A \subset X$ be locally compact and $h l c_{N}^{L}$ and let $M$ be an L-module. Then for $n \leqq N$ and $N \geqq 1$

$$
H_{n}^{c}(X, A ; M) \approx \check{H}_{n}^{c}(X, A ; M)
$$

and, if $A, X$ are both paracompact, these are isomorphic to $\check{H}_{n}(X, A ; M)$.

Proof. Again the coefficients in the Čech theory should technically be in the pair $M, M$. Also, as before, the general case follows from 
the paracompact case.

Now, on $X$, the constant cosheaf $\Phi(M)$ associated with the constant precosheaf $M$ is just $H_{0}^{c}(\cdot ; M)$ and it is locally isomorphic to $M$ (and similarly on $A$ ). Thus, by (8.12), we have

$$
\check{H}_{*}^{c}(X, A ; M) \approx \check{H}_{*}^{c}\left(X, A ; H_{0}^{c}(\cdot ; M)\right)
$$

(and similarly for the paracompact case) and the corollary follows from (9.3).

REMARK 9.5. If the base ring $L$ is a field, then the first part of (9.4) is true without the condition $h l c_{L}^{N}$ since both theories are continuous in that case. This is false for general $L$ however. Also recall that there are the implications $c l c^{N+1} \Rightarrow h l c^{N} \Rightarrow c l c^{N}$; see [1] for definitions and references.

Since writing this paper the author has discovered that (9.4) had been previously proved by Jussila in [5]. Remarks similar to (10.5) are also applicable to this case. Also see [1, 7] for related results.

10. Singular homology. For locally compact spaces we may combine the results of the preceding section with those of [1, pp. 219231] to obtain similar facts about singular homology. For general spaces we must proceed more directly.

We shall recall some constructions from [1, Chap. V]. Our approach here differs from that of [1] as regards coefficients. (Both here and in [1] the approach to coefficients is dictated by the methods used.)

Let $\mathscr{A}$ be any sheaf on the (arbitrary) space $X$. The singular chain group of $U$ in degree $n$ and with coefficients in $\mathscr{A}$ is defined by

$$
S_{n}(U ; \mathscr{A})=\bigoplus_{\sigma} \Gamma\left(\sigma^{*} \mathscr{A}\right)
$$

where the sum ranges over all singular simplices $\sigma: \Delta_{n} \rightarrow U$ of $U$ and $\sigma^{*} \mathscr{A}$ denotes the induced sheaf on $\Delta_{n}$. If $e: \Delta_{n-1} \rightarrow \Delta_{n}$ is a face map then we have an induced homomorphism

$$
\Gamma\left(\sigma^{*} \mathscr{A}\right) \longrightarrow \Gamma\left(e^{*} \sigma^{*} \mathscr{A}\right)=\Gamma\left((\sigma \circ e)^{*} \mathscr{A}\right)
$$

and it follows that the boundary operator

$$
\partial: S_{n}(U ; \mathscr{A}) \longrightarrow S_{n-1}(U ; \mathscr{A})
$$

may be defined in the usual way.

Similarly the barycentric subdivision operator

$$
s d: S_{n}(U ; \mathscr{A}) \longrightarrow S_{n}(U ; \mathscr{A})
$$


may be defined, via the continuous map $\operatorname{sd}\left(\Delta_{n}\right) \rightarrow \Delta_{n}$, and it may be shown, as usual, that $s d$ is chain homotopic to the identity. (See, for example, [10, p. 177].)

Let $S_{n}^{(i)}$ be copies of $S_{n}(U ; \mathscr{A})$ for $i \geqq 1$ and consider the direct system

$$
S_{n}^{(1)} \longrightarrow S_{n}^{(2)} \longrightarrow \cdots
$$

where the maps are the subdivision homomorphisms $s d$. We denote its direct limit by

$$
\mathfrak{S}_{n}(X ; \mathscr{A})(U)
$$

and note that the natural map

$$
S_{n}(U ; \mathscr{A})=S_{n}^{(1)} \longrightarrow \lim S_{n}^{(r)}=\mathscr{S}_{n}(X ; \mathscr{A})(U)
$$

induces an isomorphism in homology, since

$$
H_{*}\left(S_{n}^{(1)}\right) \stackrel{\approx}{\longrightarrow} H_{*}\left(S_{n}^{(r)}\right)
$$

whence

$$
H_{*}\left(S_{n}^{(1)}\right) \stackrel{\approx}{\longrightarrow} H_{*}\left(S_{n}^{(r)}\right) \approx H_{*}\left(\stackrel{\lim }{\longrightarrow} S_{n}^{(r)}\right) .
$$

The resulting (singular) homology group will be denoted by

$$
{ }_{4} H_{n}(U ; \mathscr{C}) \text {. }
$$

It is clearly the classical singular homology group when $\mathscr{A}$ is constant.

Now it is easy to see that $\mathfrak{S}_{n}(X, \mathscr{O})$ is a flabby cosheaf on $X$. (This is most easily seen by using the criterion of (1.3).)

For $A \subset X$ we have an exact sequence

$$
\mathfrak{S}_{n}(A ; \mathscr{A} \mid A)^{X}>\longrightarrow \mathfrak{S}_{n}(X ; \mathscr{A}) \longrightarrow \mathfrak{S}_{n}(X, A ; \mathscr{A})
$$

where the right hand term is defined in the same way as the absolute terms. For details on this see [1, p. 180]. By (1.1) the relative term $\mathfrak{S}_{n}(X, A ; \mathscr{A})$ is a cosheaf and it can be seen (loc. cit.) that it is flabby.

Now, by definition, we have an exact sequence

$$
\mathfrak{S}_{1}(X ; \mathscr{A}) \longrightarrow \mathfrak{S}_{0}(X ; \mathscr{A}) \longrightarrow{ }_{4} H_{0}(\cdot ; \mathscr{A}) \longrightarrow 0
$$

of precosheaves on $X$. By (1.1) it follows that ${ }_{4} H_{0}(\cdot ; \mathscr{A})$ is always a cosheaf. If $\mathscr{A}=M$ is constant then clearly ${ }_{4} H_{0}(\cdot ; M)$ is only a slight variant of the "constant cosheaf" $\mathfrak{M}$ defined in the last section. They are the same when $X$ is locally arcwise connected. For general $X$ one might call this the "singular constant cosheaf associated with $M . "$ 
Lemma 10.1. If $X$ is $H L C^{N}$ and if $\mathscr{A}$ is locally constant then $\mathfrak{S}_{*}(X: \mathscr{A})$ is a flabby $N$-coresolution of the cosheaf ${ }_{4} H_{0}(\cdot ; \mathscr{A})$.

Proof. We must show that the precosheaf ${ }_{4} H_{n}(\cdot ; \mathscr{A})$ is locally zero for $0<n \leqq N$. For $\mathscr{A}=Z$ this is the definition of the property $H L C^{N}$, and for constant it follows from the universal coefficient theorem. The locally constant case obviously follows from the constant case.

From (10.1) and (8.10) we obtain immediately:

Theorem 10.2. Let $A \subset X$ both be paracompact and $H L C^{N}$. Let $\mathscr{A}$ be a locally constant sheaf on $X$. Then there is a canonical isomorphism for $n \leqq N$ :

$$
{ }_{4} H_{n}(X, A ; \mathscr{A}) \approx \check{H}_{n}\left(X, A ;{ }_{4} H_{0}(\cdot ; \mathscr{A})\right) .
$$

Similarly, from (8.11) we obtain:

CoRollary 10.3. Let $A \subset X$ both be locally compact and $H L C^{N}$. Let $\mathscr{A}$ be a locally constant sheaf on $X$. Then for $n \leqq N$

$$
{ }_{\Delta} H_{n}(X, A ; \mathscr{A}) \approx \check{H}_{n}^{c}\left(X, A ;{ }_{\Delta} H_{0}(\cdot ; \mathscr{A})\right) .
$$

Again the following result is proved in the same manner as is (9.4):

Corollary 10.4. Let $A \subset X$ both be paracompact (respectively, locally compact) and $H L C^{N}$. Let $M$ be any abelian group. Then for $n \leqq N$

$$
{ }_{4} H_{n}(X, A ; M) \approx \check{H}_{n}(X, A ; M)
$$

(respectively, $\left.\approx \check{H}_{n}^{c}(X, A ; M)\right)$.

REMARK 10.5. This latter result was first proved by Mardešić [8] who proved the isomorphism under the slightly weaker hopothesis in the paracompact case that $A$ and $X$ are $H L C^{V-1}$ and have neighborhood bases projecting homologically trivially into the total space in degree $N$ (singular homology). The present proof can also easily be extended to this case but we have chosen, for simplicity of terminology not to do this. (We only note that to achieve the extension one takes the original covering $\mathfrak{U}$ in the proof of (7.1) to be $\{X\}$.) The reader should note that when the present proof is stripped of all the nonessential material it is, in fact, a very efficient proof which avoids the intricate refinement arguments of [8]. Essentially, these intricate details are subsumed in the general theory. 
11. Acyclic coverings. In this section we shall confine our attention to absolute homology.

Let $\mathfrak{A}_{*}$ be a flabby differential cosheaf with nonnegative degrees and let $\mathfrak{A}=\operatorname{Coker}\left\{\mathfrak{U}_{1} \rightarrow \mathfrak{A}_{0}\right\}$. Consider the spectral sequence (6.1) for a covering $\mathfrak{U}$ of $X$ :

$$
E_{p, q}^{2}(\mathfrak{U})=\check{H}_{p}\left(\mathfrak{U} ; \mathfrak{S}_{q}\left(\mathfrak{A}_{*}\right)\right) \Longrightarrow H_{p+q}\left(\mathfrak{A}_{*}(X)\right) .
$$

We have the canonical edge homomorphism

$$
\lambda \mathfrak{u}: H_{n}\left(\mathfrak{A}_{*}(X)\right) \longrightarrow \check{H}_{n}(\mathfrak{U} ; \mathfrak{A}) \text {. }
$$

The following result is immediate:

THEOREm 11.1. If each finite intersection $U$ of members of $\mathfrak{u}$ has $H_{q}\left(\mathfrak{A}_{*}(U)\right)=0$ for $q \neq 0$, then $\lambda_{\mathfrak{u}}$ is an isomorphism in each degree.

For Borel-Moore homology we have

CoRollary 11.2. Let $\mathfrak{U}$ be a covering of the locally compact and locally connected space $X$ such that for each finite intersection $U$ of members of $\mathfrak{u}$ we have $H_{q}^{c}(U ; \mathscr{A})=0$ for $q>0$, where $\mathscr{A}$ is some given sheaf on $X$. Then there is a canonical isomorphism

$$
H_{n}^{c}(X ; \mathscr{A}) \approx \check{H}_{n}\left(\mathfrak{U} ; H_{0}^{c}(\cdot ; \mathscr{A})\right) .
$$

For singular homology we have

CoRollary 11.3. Let $\mathfrak{U}$ be a covering of the space $X$ such that for each finite intersection $U$ of members of $\mathfrak{H}$ we have ${ }_{4} H_{q}(U ; \mathscr{A})=0$ for $q>0$, where $\mathscr{A}$ is some given sheaf on $X$. Then there is a canonical isomorphism

$$
{ }_{4} H_{n}(X ; \mathscr{A}) \approx \check{H}_{n}\left(\mathfrak{U} ;{ }_{4} H_{0}(\cdot ; \mathscr{A})\right) .
$$

12. Applications to maps. In this section we shall consider a continuous map

$$
f: E \longrightarrow X .
$$

For a precosheaf $\mathfrak{B}$ on $E$ we define its direct image $f \mathfrak{B}$ on $X$ by $(f \mathfrak{B})(U)=\mathfrak{B}\left(f^{-1} U\right)$ (see [1, p. 179]). Clearly $f \mathfrak{B}$ is a cosheaf when $\mathfrak{B}$ is and is flabby when $\mathfrak{B}$ is. If $\mathfrak{B}_{*}$ is a flabby differential cosheaf on $E$ then $\mathfrak{A}_{*}=f \mathfrak{B}_{*}$ is one on $X$. Thus the spectral sequence of (6.1) has 


$$
E_{p, q}^{2}(\mathfrak{M})=\check{H}_{p}\left(\mathfrak{U} ; f \mathfrak{W}_{q}\left(\mathfrak{B}^{*}\right)\right) \Longrightarrow H_{p+q}\left(\mathfrak{B}_{*}(X)\right)
$$

natural in coverings $\mathfrak{U}$ of $X$.

In particular, for Borel-Moore homology (where $E$ is locally compact) we have spectral sequences

$$
E_{p, q}^{2}(\mathfrak{U})=\check{H}_{p}\left(\mathfrak{U} ; H_{q}^{c}\left(f^{-1}(\cdot) ; \mathscr{A}\right)\right) \Longrightarrow H_{p+q}^{c}(E ; \mathscr{A})
$$

natural in coverings $\mathfrak{U}$ of $X$ and sheaves $\mathscr{A}$ on $E$.

Similarly, for singular homology, we have spectral sequences

$$
E_{p, q}^{2}(\mathfrak{u})=\check{H}_{p}\left(\mathfrak{u} ;{ }_{\Delta} H_{q}\left(f^{-1}(\cdot) ; \mathscr{C}\right)\right) \Longrightarrow{ }_{\Delta} H_{p+q}(E ; \mathscr{A})
$$

natural in coverings $\mathfrak{U}$ of $X$ and sheaves $\mathscr{A}$ on $E$.

We shall now prove a generalization of (7.2). Suppose that $\mathfrak{A}_{*}$ is a flabby differential cosheaf with $\mathfrak{A}_{n}=0$ for $n<0$ (or generally with degrees bounded below). Suppose, moreover that we are given integers $0 \leqq k<N$ such that

$$
\mathfrak{g}_{q}\left(\mathfrak{A}^{*}\right) \text { is locally zero for all } q \neq k, q \leqq N \text {. }
$$

Let $\mathfrak{Z}_{n}=\operatorname{Ker}\left\{d_{n}: \mathfrak{A}_{n} \rightarrow \mathfrak{A}_{n-1}\right\}$. Then by $(2.3)$

$$
\mathfrak{A}_{k} \longrightarrow \mathfrak{A}_{k-1} \longrightarrow \cdots \longrightarrow \mathfrak{A}_{0} \longrightarrow 0
$$

is exact and

$$
3_{k} \text { is a flabby cosheaf. }
$$

Moreover, it is clear that

$$
\mathfrak{A}_{N+1} \longrightarrow \cdots \longrightarrow \mathfrak{A}_{k+1} \longrightarrow \mathfrak{3}_{k} \longrightarrow \mathfrak{S}_{k}\left(\mathfrak{A}_{*}\right) \longrightarrow 0
$$

is locally exact. Thus, under the hypothesis (12.1), we see that $\mathfrak{S}_{k}\left(\mathfrak{U}_{*}\right)$ is a cosheaf and that the differential cosheaf $\mathfrak{Y}_{*}^{\prime}$, defined by

$$
\mathfrak{A}_{q}^{\prime}= \begin{cases}0 & q<0 \\ \mathfrak{3}_{k} & q=0 \\ \mathfrak{A}_{k+q} & q>0\end{cases}
$$

is a flabby $(N-k)$-coresolution of $\mathfrak{S}_{k}\left(\mathfrak{A}_{*}\right)$.

By (7.2) [and (8.11) in the absolute case] we obtain:

THEOREM 12.2. Let $X$ be paracompact (respectively, locally compact) and let $\mathfrak{H}_{*}$ be a flabby differential cosheaf on $X$ with degrees bounded below such that (12.1) is satisfied. Then there is the canonical isomorphism 


$$
H_{n}\left(\mathfrak{A}_{*}(X)\right) \approx \check{H}_{n-k}\left(X ; \mathfrak{S}_{k}\left(\mathfrak{A}_{*}\right)\right)
$$

for $n \leqq N . \quad$ (Respectively, with $\check{H}_{n-k}^{c}$ in place of $\check{H}_{n-k}$.)

Now we return to the discussion of a map $f: E \rightarrow X$ and we let $E_{0} \subset E$ be any subspace. Applying (12.2) to the flabby differential cosheaf $f \mathfrak{C}_{*}^{c}\left(E, E_{0} ; \mathscr{A}\right)$ we obtain:

Corollary 12.3. Suppose that $X$ and $\left(E, E_{0}\right)$ are locally compact, that $\mathscr{A}$ is some sheaf on $E$ and that there are integers $k<N$ such that for $q \leqq N, q \neq k$ we have that the precosheaf

$$
H_{q}^{c}\left(f^{-1}(\cdot), f^{-1}(\cdot) \cap E_{0} ; \mathscr{A}\right)
$$

on $X$ is locally zero. Then this precosheaf is zero for $q<k$ and is a cosheaf for $q=k$. Moreover, we have a canonical isomorphism

$$
H_{n}^{c}\left(E, E_{0} ; \mathscr{A}\right) \approx \check{H}_{n-k}^{c}\left(X ; H_{k}^{c}\left(f^{-1}(\cdot), f^{-1}(\cdot) \cap E_{0} ; \mathscr{A}\right)\right) .
$$

for $n \leqq N$.

Similarly applying (12.2) to $f \mathfrak{S}_{*}\left(E, E_{0} ; \mathscr{A}\right)$ we have

COROLlary 12.4. Suppose that $X$ is paracompact (respectively, locally compact), that $\mathscr{A}$ is some sheaf on $E$ and that there are integers $k<N$ such that for $q \leqq N, q \neq k$ we have that the precosheaf ${ }_{\triangle} H_{q}\left(f^{-1}(\cdot), f^{-1}(\cdot) \cap E_{0} ; \mathscr{A}\right)$ on $X$ is locally zero. Then this precosheaf is zero for $q<k$ and is a cosheaf for $q=k$. Moreover, there is a canonical isomorphism

$$
{ }_{\triangle} H_{n}\left(E, E_{0} ; \mathscr{A}\right) \approx \check{H}_{n-k}\left(X ;{ }_{4} H_{k}\left(f^{-1}(\cdot), f^{-1}(\cdot) \cap E_{0} ; \mathscr{A}\right)\right)
$$

for $n \leqq N$ (respectively, with $\check{H}_{n-k}^{c}$ replacing $\check{H}_{n-k}$ ).

\section{REFERENCES}

1. G. E. Bredon, Sheaf Theory, McGraw-Hill, 1967.

2. R. Deheuvels, Homologie des ensembles ordonnés et des espaces topologiques, Bull. Soc. Math. de France 90 (1962), 261-321.

3. P. Freyd, Abelian Categories, Harper and Row, 1964.

4. P. Gabriel and M. Zisman, Calculus of Fractions and Homotopy Theory, SpringerVerlag, 1967.

5. O. Jussila, On homology theories in locally connected spaces, II, Ann. Acad. Sci. Fenn. (A) 378 (1965), 8pp.

6. J. W. Gray, Sheaves with values in a category, Topology 3 (1964), 1-18.

7. C. N. Lee, The regular convergence theorem, Mich. Math. J. 14 (1967), 207-217.

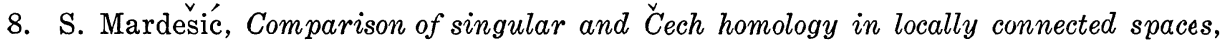
Mich. Math. J. 6 (1959), 151-166. 
9. B. Mitchell, Theory of Categories, Academic Press, 1965.

10. E. H. Spanier, Algebraic Topology, McGraw-Hill, 1966.

Received February 8, 1967. Supported in part by National Science Foundation grant GP-3990 and by a Sloan fellowship.

University of California, Berkeley and

The Institute For Advanced Study 


\section{PACIFIC JOURNAL OF MATHEMATICS}

\section{EDITORS}

H. ROYDEN

Stanford University

Stanford, California

\section{J. P. Jans}

University of Washington

Seattle, Washington 98105

\section{J. DUGUNDJI}

Department of Mathematics University of Southern California Los Angeles, California 90007

\section{RICHARD ARENS}

University of California

Los Angeles, California 90024

\section{ASSOCIATE EDITORS}

E. F. BECKENBACH

B. H. NEUManN

F. WOLF

K. YosIDA

\section{SUPPORTING INSTITUTIONS}

UNIVERSITY OF BRITISH COLUMBIA CALIFORNIA INSTITUTE OF TECHNOLOGY UNIVERSITY OF CALIFORNIA MONTANA STATE UNIVERSITY UNIVERSITY OF NEVADA NEW MEXICO STATE UNIVERSITY OREGON STATE UNIVERSITY UNIVERSITY OF OREGON OSAKA UNIVERSITY UNIVERSITY OF SOUTHERN CALIFORNIA
STANFORD UNIVERSITY

UNIVERSITY OF TOKYO UNIVERSITY OF UTAH WASHINGTON STATE UNIVERSITY UNIVERSITY OF WASHINGTON AMERICAN MATHEMATICAL SOCIETY CHEVRON RESEARCH CORPORATION TRW SYSTEMS NAVAL WEAPONS CENTER 


\section{Pacific Journal of Mathematics}

\section{Vol. 25, No. $1 \quad$ September, 1968}

Glen Eugene Bredon, Cosheaves and homology................... 1

Robin Ward Chaney, A chain rule for the transformation of integrals in

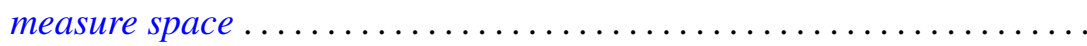

Colin W. Clark, On relatively bounded perturbations of ordinary differential operators................................... 59

John Edwin Diem, A radical for lattice-ordered rings.............. 71

Zeev Ditzian, On a class of convolution transforms ................ 83

Dennis Garoutte and Paul Adrian Nickel, A note on extremal properties characterizing weakly $\lambda$-valent principal functions............. 109

Shwu-Yeng Tzeng Lin, Fixed point properties and inverse limit spaces . . . 117

John S. Lowndes, Some dual series equations involving Laguerre polynomials ................................. 123

Kirti K. Oberai, Sum and product of commuting spectral operators ....... 129

J. N. Pandey and Armen H. Zemanian, Complex inversion for the generalized convolution transformation..................... 147

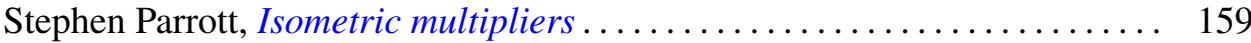

Manoranjan Prasad, Note on an extreme form .................. 167

Maciej Skwarczyński, A representation of a bounded function as infinite product in a domain with Bergman-Shilov boundary surface ......... 177

John C. Taylor, The Šilov boundary for a lattice-ordered semigroup ...... 185 Donald Reginald Traylor and James Newton Younglove, On normality and pointwise paracompactness ............................. 193

L. Tzafriri, Quasi-similarity for spectral operators on Banach spaces ... 